\title{
Orientation des monastères mongols
}

The orientation of Mongolian monasteries

\author{
Isabelle Charleux
}

\section{(2) OpenEdition}

Journals

Édition électronique

URL : https://journals.openedition.org/emscat/724

DOI : $10.4000 /$ emscat.724

ISSN : 2101-0013

Éditeur

Centre d'Etudes Mongoles \& Sibériennes / École Pratique des Hautes Études

Édition imprimée

Date de publication : 1 mai 2006

Pagination : 239-281

ISBN : 2-9518888-3-X

ISSN : 0766-5075

Référence électronique

Isabelle Charleux, "Orientation des monastères mongols », Études mongoles et sibériennes, centrasiatiques et tibétaines [En ligne], 36-37 | 2006, mis en ligne le 17 mars 2009, consulté le 13 juillet 2021. URL : http://journals.openedition.org/emscat/724 ; DOl : https://doi.org/10.4000/emscat.724

Ce document a été généré automatiquement le 13 juillet 2021.

(c) Tous droits réservés 


\title{
Orientation des monastères mongols
}

The orientation of Mongolian monasteries

\author{
Isabelle Charleux
}

1 Entre le $\mathrm{xvI}^{\mathrm{e}}$ et le $\mathrm{xx}^{\mathrm{e}}$ siècle, les Mongols, convertis au bouddhisme tibétain, ont fondé quelque deux mille temples et monastères qui ont transformé le paysage et créé une nouvelle géographie sacrée souvent superposée à la géographie de l'époque prébouddhique.Toutefois, les Mongols n'ont pas adopté en bloc la vision tibétaine du monde en faisant table rase de leurs représentations anciennes. D'autre part, les influences de la cosmologie chinoise, déjà présente dans les steppes avant notre ère et qui imprègne la vie matérielle et spirituelle des Mongols depuis le XIII ${ }^{e}$ siècle, se font d'autant plus ressentir dans l'architecture que les charpentiers travaillant en Mongolie sont souvent des Chinois.

2 Comment les monastères mongols occupent-ils l'espace et s'y positionnent-ils, comment s'orientent-ils, et dans quelles directions se développent-ils? Quels sont les facteurs qui déterminent l'orientation des monastères et des bâtiments qui les composent, et celle-ci revêt-elle une importance symbolique particulière ? Dans quelle mesure l'orientation et la conception de l'espace religieux autochtones ont-elles influencé cette architecture d'origine étrangère ? En considérant à la fois l'inscription du monastère dans le paysage et son organisation propre, le présent article se propose d'étudier l'importance de l'orientation dans le choix d'un site, l'éventuel modèle cosmologique qui la sous-tend, et l'adéquation entre la réalité et la représentation littéraire ou artistique qui en est faite. Les questions de géomancie, de représentation de l'espace, de hiérarchie entre différentes positions (droite et gauche, haut et bas, entrée et fond) et d'exigences de la règle monastique, du culte et des rituels permettent d'expliquer en partie l'emplacement et l'orientation des bâtiments d'un monastère. En dernier lieu sera évoquée la pratique de la circumambulation, qui unit en un même mouvement parcours spatial et parcours temporel.

3 Les pratiques architecturales des Mongols à l'époque moderne n'ayant encore fait l'objet que de rares publications, cet article se fonde essentiellement sur des 
observations personnelles lors de recherches en Mongolie-Intérieure et en république de Mongolie ${ }^{1}$ entre 1993 et 2002.

\section{Direction générale vers laquelle s'orientent les monastères mongols}

4 Un premier constat sur l'orientation des monastères mongols peut être déduit des édifices préservés : les architectures privilégient généralement une orientation vers le sud. Les portes des enceintes ouvrent au sud, les façades des principaux temples sont orientées vers le sud, alors que les temples latéraux, d'importance moindre, sont ouverts vers le centre du monastère, vers l'est ou vers l'ouest.

5 Toutefois, une minorité de monastères mongols s'orientent vers l'est, vers une direction intermédiaire entre sud et est ou, plus rarement, vers l'ouest. Dans certains cas, le choix de la direction est tributaire de l'orientation de la pente du terrain ou de la montagne, comme pour leSir-a mören süm-e (dans la bannière des Dörben keüked [chinois: Siziwang], ligue d'Ulayancabu, Mongolie-Intérieure), situé au pied d'une chaîne de collines nord-sud, qui ouvre vers l'est, ou pour le Gilubar zuu (dans la bannière de gauche des Baүarin, municipalité d'Ulayanqada [chinois : Chifeng], M.-I.), adossé à un immense rocher, tourné vers le sud-ouest. D'autres monastères, pourtant situés dans la steppe, n'ouvrent pas vers le sud : le Jeke zuu (Vang-un youl-un zuu, dans la bannière Dalad, ligue des Ordos ou du Jeke zuu, M.-I.) est orienté vers l'est ; le Zuuqajin süm-e (dans la bannière Otuy de l'avant, ligue des Ordos, M.-I.), le Bandida gegen süm-e (dans la ville de Sili-jin qota, ligue de Sili-jin youl, M.-I.) et le Gegen süm-e (dans la bannière de l'avant de l'aile droite des Qorcin, ligue de Kingyan [chinois : Xing'an], M.I.) font face au sud-est.

6 La faveur accordée au sud n'est pas surprenante : les Mongols orientent généralement l'entrée de leur yourte ou de leur maison (quand cela leur est possible) ${ }^{2}$ vers le sud, ce qui est attesté à l'époque des fondations bouddhiques, entre le $\mathrm{xvI}^{\mathrm{e}}$ et le $\mathrm{xx}^{\mathrm{e}}$ siècle ${ }^{3}$. Le sud était déjà semble-t-il l'orientation généralement adoptée par les Mongols de l'empire gengiskhanide ${ }^{4}$. Les Mongols Ordos et certains Mongols occidentaux, toutefois, préfèrent ouvrir leurs habitations vers l'est ${ }^{5}$ ou le sud-est. Hommage au lever du soleil, l'est semble être l'ancienne orientation commune aux peuples de la steppe, d'après ce que l'archéologie et les sources écrites nous révèlent ${ }^{6}$.

7 L'architecture chinoise, qui inspira nombre de monastères mongols, a choisi le sud comme orientation privilégiée depuis une haute antiquité. Cette orientation est liée à la valorisation du yang (côté exposé au soleil) ainsi qu'à l'ensemble de la cosmologie. Le dieu, comme l'empereur, trône face au sud dans son palais. De même, depuis le second millénaire, les temples tibétains privilégient le sud comme orientation de référence (chayet 1994, pp. 125 et 143-152). L'architecture des monastères gelugpa ${ }^{7}$, issue d'un pouvoir centralisé qui conquit l'ensemble de l'aire tibétaine et pénétra en Mongolie au début du XVII ${ }^{e}$ siècle, généralisa l'ouverture des temples vers le sud. L'usage ancien d'ouvrir les temples à l'est ${ }^{8}$ ou au sud-est ne fut cependant pas totalement abandonné. La configuration du terrain faisait fréquemment choisir des orientations différentes du sud ou de l'est: par exemple les temples du monastère de Kumbum ${ }^{9}$ (région du Koukounor, $\mathrm{Amdo}^{10}$ ), qui fut une source d'inspiration tant en matière d'enseignement 
que d'architecture pour de nombreux monastères mongols, font face à l'est, au sud-est, au sud-ouest, certains au nord ou à l'ouest.

De même, en Mongolie, l'orientation du monastère déviera du sud idéal selon les caractéristiques du terrain, ou selon la rose des vents, donnée prioritaire dans le choix de l'orientation. Dans une grande partie de la Mongolie, les vents les plus violents viennent du nord-ouest, mais il existe selon les lieux des vents dominants du sud, de l'est, etc.: on cherchera, pour la yourte, la maison et le temple, à adosser une construction contre le vent et à diriger la porte de sorte à obtenir un ensoleillement maximal.

Le visiteur muni d'une boussole constate que « le sud » est pris au sens large. L'orientation vers l'un des points cardinaux est souvent approximative, déviant de quelques degrés de l'axe. Les connaissances actuelles ne permettent pas de savoir si des calculs astrologiques précis étaient effectués ou si une boussole était employée pour déterminer le sud exact lors de la fondation d'un monastère. Il semble en tout cas que l'on ne trouve pas en Mongolie un souci de calcul aussi précis de l'orient exact que dans le monde indien ${ }^{11}$. Les Mongols semblent voir les orients plus comme une direction générale que comme un point particulier ${ }^{12}$.

10 Les récits de fondation et les moines interrogés révèlent que l'orientation générale n'est qu'un paramètre, un critère parmi d'autres dans le choix de l'implantation d'un monastère. Quelques données sur la façon dont un lama voulant bâtir un nouveau monastère cherchait un site propice nous renseignent sur l'importance accordée à l'orientation.

\section{Le choix d'un site}

11 Le canon bouddhique ne donne pas de recommandation particulière en matière d'orientation. En Mongolie, les fondateurs avaient quasiment toute liberté de choisir un site, du moment qu'aucun dommage n'était causé à d'éventuels agriculteurs. L'étude de récits de fondation montre que trois facteurs majeurs déterminaient le choix d'un site: la volonté ou non d'isolement de la communauté monastique, les facteurs environnementaux (géographiques et climatiques) et les règles de l'ensemble de pratiques aux origines diverses généralement nommé « géomancie ».

12 L'établissement d'un monastère à proximité ou à distance des centres urbains, des routes commerciales et des parcours de nomadisation était lié à la dépendance du monastère par rapport à sa source de financement et à son idéal contemplatif : un monastère qui vivait des donations de ses fidèles nomadisait avec eux ou recherchait un lieu de passage, un point d'eau fréquenté, tandis qu'un ermitage ou un monastère comprenant des facultés monastiques et disposant de ressources propres pouvait choisir un site peu ou pas fréquenté. Quelle qu'ait été la densité humaine recherchée, les monastères possédaient des troupeaux et devaient leur assurer de bons pâturages pour l'été et l'hiver.

13 On constate que dans la durée, le degré d'isolement n'est pas vraiment constant, car un monastère, même isolé à l'origine, jouait un rôle centripète, attirant à lui éleveurs, marchands et pèlerins et détournant vers lui les routes caravanières de leur tracé ancien, si sa renommée dépassait les frontières de la bannière. Comme le montrent les cartes de bannières dessinées au XIX ${ }^{e}$ et au XX $X^{e}$ siècle, le monastère construit en dur ${ }^{13}$ 
était souvent le seul point fixe dans un monde encore essentiellement nomade (bien que restreint par les frontières des bannières instaurées par les Qing ${ }^{14}$. À la fin du $\mathrm{XIX}^{\mathrm{e}}$ siècle, des bourgs se formèrent progressivement autour des monastères, lorsque les commerçants chinois, les charpentiers et les šabi (sujets laïques administrativement rattachés à un monastère ou à un lama réincarné) se sédentarisaient à proximité. Ainsi, le monastère s'orientait, au moment de sa fondation, par référence aux groupes humains ; par la suite ce sont les hommes qui s'orientèrent par rapport à lui, le prenant comme point de repère et s'y rassemblant pendant les grands festivals.

Certains monastères furent bâtis en ville, ou, dans la steppe, à proximité d'un palais ou d'un campement princier, tels les temples de bannière édifiés par les princes dirigeant une bannière mongole (zasay). Leur fondation obéissait à une règle: le complexe religieux n'était jamais construit derrière la résidence, c'est-à-dire à son nord lorsqu'elle ouvrait vers le sud. Les trois grands monastères de Kökeqota furent bâtis au $\mathrm{XVI}^{\mathrm{e}}$ et au début du XVII ${ }^{\mathrm{e}}$ siècle au sud du palais d'Altan Qan, en accord avec les principes urbanistiques chinois. Le Yanfu si, temple princier de la bannière Alašan de gauche (M.-I.), fut construit en 1733 à l'ouest de la résidence du prince, dans la ville de Dingyuanying (actuelle Bajanqota). Comme nous le verrons dans la suite de cet exposé, les bâtiments construits au nord étant plus "hauts " que les bâtiments au sud, le monastère ou le temple princier peut être considéré par rapport au palais comme subordonné ou sur le même plan.

Quelques monastères recherchèrent la proximité de ruines d'anciennes villes afin d'en remployer les matériaux: ainsi Erdeni zuu jouxte le mur sud de l'ancienne ville de Qaraqorum (province d'Övörkhangaï, république de Mongolie), et plusieurs monastères s'établirent près de villes kitan (comme Qingzhou, dans la bannière de droite des Bayarin, municipalité d'Ulayanqada [chinois : Chifeng], M.-I.). Cependant, pour ne pas avoir à déblayer (car ces villes étaient toutes ruinées, à l'exception parfois d'une pagode kitan encore debout) et par crainte que les esprits de la ville morte ne vinssent hanter les vivants, l'on évitait de bâtir sur les ruines mêmes.

\section{Le fantasme de la montagne}

Dans la majorité des cas, les monastères s'implantaient sur un territoire vierge de toute construction, en tentant de s'adapter à la configuration du terrain et même de l'« exploiter ». Les fondateurs furent particulièrement attirés par les montagnes, qui protégeaient contre le vent et offraient de riches pâturages d'hiver. L'attrait de la montagne s'explique aussi par son importance dans la géomancie (rôle de barrière contre les influences malignes venues du nord), par la vision du cosmos bouddhique (l'univers ordonné autour du mont Sumeru) ${ }^{15}$ et par son rôle dans la religion prébouddhique des Mongols, puisqu'elle est supposée abriter les mânes des ancêtres. Les moines bouddhistes ont, consciemment ou non, cherché à récupérer cette ancienne géographie sacrée en marquant de temples les points stratégiques, de même qu'ils s'investissaient dans le culte des esprits locaux aux obos (obup-a;qalq-a: ovoo) et intégraient la divinité du lieu parmi les dieux protecteurs de leur panthéon.

17 Les monastères cherchant la proximité de voies de communication s'implantaient au pied d'une montagne, ceux qui voulaient s'isoler cherchaient un site plus difficile d'accès, par exemple un cirque couronné par des sommets et protégé par un étroit défilé, ou un fond de vallée (comme le Barayun keid et le Zegün keid de la bannière 
Alašan de gauche et le Badyar coiling süm-e à l'est de Buүutu [chinois : Baotou], M.-I.). Les sites rupestres, qui occupent une place importante dans la tradition bouddhique, étaient particulièrement recherchés.

Toutefois, compte tenu du relief de la Mongolie, beaucoup de monastères à la recherche d'un site montagneux devaient se contenter des petites collines ou des soulèvements vallonnés coupés par de brusques failles de la steppe mongole. Nombre d'entre eux sont adossés à une colline ou situés sur une très légère pente (le Sir-a mören süm-e de la bannière des Dörben keüked et les deux grands monastères Köke süm-e et Sir-a süm-e de Dolonnor, en Mongolie-Intérieure; l'Amurbajasqulangtu keid, dans la province de Sélenga, en république de Mongolie) ou en terrain plat (Erdeni zuu). Or, les monastères situés en terrain plat ou légèrement pentu sont décrits dans les sources ou représentés en peinture sur le versant d'une montagne. C'est par exemple le cas du Fanzong si (dans la bannière des Ongniyud, municipalité d'Ulayanqada [chinois : Chifeng], M.-I.), décrit par une monographie locale dans un site montagneux : des collines (qui ne sont mêmes pas visibles depuis le monastère) se trouvent à une vingtaine de kilomètres au nord. Le Köndelen zuu et d'autres monastères de la région de Buүutu [chinois: Baotou], en Mongolie-Intérieure, sont représentés dans une peinture au pied de hautes montagnes (fig. 1), celles-ci étant en fait des collines situées à plusieurs kilomètres des bâtiments. La montagne est souvent plus un idéal qu'une réalité, et le monastère se situe par rapport à elle en masquant, dans son discours, la distance qui l'en sépare.

\section{La géomancie}

19 L'orientation dépend aussi des exigences de l'examen des caractéristiques du terrain, désigné en français sous le terme de " géomancie ", et dont la montagne n'est qu'une des prescriptions.Avant toute construction bouddhique, le site est toujours minutieusement choisi en fonction de ses caractéristiques favorables ou néfastes. Ces pratiques " géomantiques ", mal documentées pour la Mongolie, sont de toute évidence inspirées du fengshui chinois, des pratiques tibétaines (qui reçurent elles-mêmes une influence chinoise) et des critères de choix des campements d'été et d'hiver des éleveurs mongols. Ces derniers ne sauraient être qualifiés de tradition autochtone de géomancie - on parlerait plutôt d'un savoir pratique des pasteurs transmis de génération en génération ${ }^{16}$. 
fig 1. - Dessin représentant le Köndelen zuu (Heissig 1959, pl. VII)

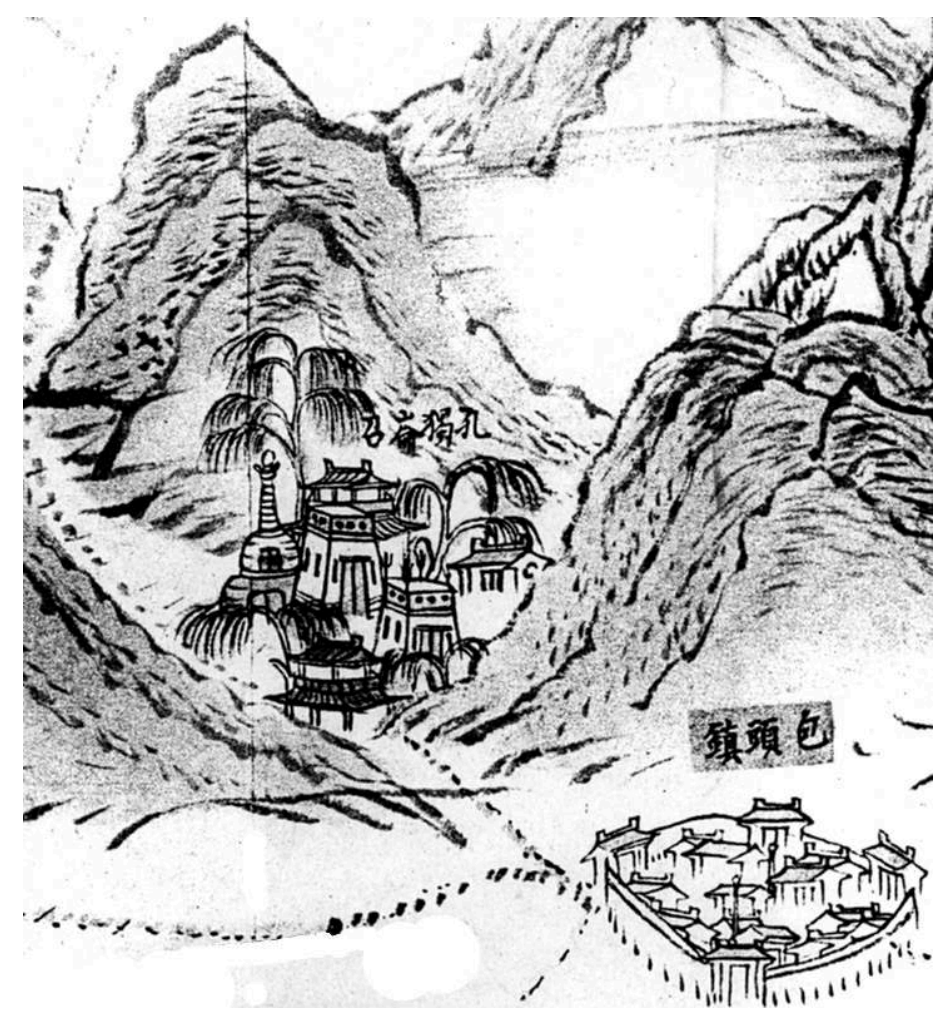

Nous ne connaissons les pratiques géomantiques appliquées en Mongolie qu'à travers quelques récits de fondation et de rares ouvrages. Aleksei Pozdneev ([1887] 1978, pp. 49-53), par exemple, nous livre un des rares témoignages détaillés, bien qu'indirect, du choix d'un site selon les prescriptions tibétaines en Mongolie Qalq-a. D'autres sources viennent confirmer ses observations : l'examen des caractéristiques du terrain était généralement réalisé par un moine "astrologue » (ziruqaici),diplômé d'un collège de Kālacakra (Doingqur dacang), qui étudiait la tradition géomantique tibétaine (sa rtags dpyad ou sa dpyad, « examen du sol») introduite en Mongolie à la fin du XVI e siècle. Les ziruqaici étaient également sollicités par les laïcs ${ }^{17}$. Ils se fondaient sur des textes tibétains du canon bouddhique; on sait également que les disciples du grand traducteur oïrate Zaja pandita traduisirent un livre de géomancie du tibétain en mongol (Bawden 1961b, p. 228 note 42). Toutefois, il n'est pas possible de dire si les moines mongols ont appliqué les préceptes tibétains à la lettre ou bien s'ils en ont fait des adaptations qui tenaient compte de pratiques locales transmises oralement ou de l'influence du fengshui.L'astrologie chinoise était enseignée conjointement avec l'astrologie tibétaine dans deux collèges du grand monastère de Labrang ${ }^{18}$ en Amdo (dans l'actuel Gansu), qui entretenait des liens étroits avec des monastères mongols; il est possible que le fengshui se soit également introduit dans les collèges bouddhiques de Mongolie.

En Mongolie méridionale, le moine " astrologue » n'était pas le seul habilité à pratiquer la géomancie: il était concurrencé par les spécialistes chinois de fengshui ${ }^{19}$, les charpentiers eux-mêmes et les chamanes. Dans l'est de la Mongolie-Intérieure, il était courant de faire appel à un géomancien chinois (shishi) pour la fondation d'un monastère, surtout s'il était célèbre - preuve supplémentaire de l'équivalence des deux systèmes pour les Mongols ${ }^{20}$. Par exemple, en 1680, le fondateur du monastère de 
Šongqur (dans la bannière de l'arrière de l'aile gauche des Qorcin, ligue de Zirim) fit venir de Pékin un géomancien réputé (Erihen 1986, p.79). Lorsque les Mongols méridionaux commencèrent à se faire construire des maisons, des palais et des temples à partir du XVI ${ }^{e}$ siècle, les charpentiers étaient majoritairement chinois, et faisaient intervenir un spécialiste de fengshui, ou avaient eux-mêmes des notions de géomancie, ce qui permettait de diminuer les coûts de construction ${ }^{21}$. C'est ainsi que les Mongols méridionaux, sans doute plus que les Qalq-a, ont été perméables aux traditions géomantiques chinoises.

Il existe quelques manuels mongols - moins d'une dizaine est aujourd'hui connue -, qui sont des traductions, extraits ou compilations d'ouvrages chinois. Aucun n'est consacré spécifiquement à l'architecture religieuse, mais certains donnent des prescriptions également valables pour la fondation de temples. Bien que la géomancie soit considérée comme une branche de la divination (de même que l'astrologie s'occupe de connaître le moment approprié de chaque événement important de la vie, la géomancie détermine l'endroit propice où il doit prendre place), ces rares ouvrages sont beaucoup moins nombreux et populaires chez les Mongols que les ouvrages d'astrologie et de divination. On peut citer notamment le Tazar-un sinzi-jin sudur [Traité des caractéristiques du terrain $]^{22}$, traduction mongole d'un manuel chinois, et deux ouvrages illustrés de diagrammes présentant les caractéristiques topographiques, mêlant traditions mongoles, chinoises et tibétaines ${ }^{23}$. Six autres ouvrages ou parties d'ouvrages connus peuvent être inclus dans ce type de littérature (Sarközi 1976, pp. 584-585). Alice Sarközi (1976) a traduit et commenté un ouvrage de géomancie de sept pages, probablement d'origine oïrate, traduit du chinois par un lama, mais fortement influencé par des traditions mongoles ${ }^{24}$.

Ces manuels en mongol donnent des instructions pour construire une maison, un temple, établir un campement et creuser une tombe. Ils expliquent comment examiner la terre, la forme et la couleur des montagnes, la direction des rivières et des routes, les traces des courants locaux influençant l'atmosphère d'un site. Si le site choisi ne s'harmonise pas avec ces courants, les habitants en subiront les effets néfastes. Au contraire si le site est favorable, les habitants connaîtront richesse, santé, longévité et bonheur. En reprenant la tradition tibétaine selon laquelle la géomancie chinoise aurait été introduite au Tibet au VII ${ }^{e}$ siècle par l'épouse chinoise du roi Songtsen Gampo ${ }^{25}$, deux de ces textes ${ }^{26}$ suggèrent que les Mongols pensaient qu'il s'agissait d'une seule et même tradition ${ }^{27}$. À cette époque où la culture mongole s'imprégnait d'influences tibétaines et chinoises, les prenait avec éclectisme et les assimilait, les systèmes chinois et tibétains, qui ne comportent pas de contradiction majeure dans leurs prescriptions générales, semblent avoir été considérés comme équivalents.

\section{De la théorie à la pratique : quelques exemples}

Sans détailler les différences entre le fengshui et la géomancie tibétaine ${ }^{28}$, contentonsnous de mentionner quelques prescriptions générales communes aux deux systèmes. Des critères pratiques se mêlent à des considérations symboliques ou sont justifiés par des explications symboliques : proximité d'un cours d'eau, de pâturages de qualité ou d'une forêt pour les matériaux de construction, site abrité. Le monastère idéal est bâti sur un terrain pentu orienté vers le sud, au pied d'une montagne "protectrice " à laquelle il s'adosse, et devant laquelle coulent un ou plusieurs cours d'eau. L'orientation 
apparaît comme une des caractéristiques principales de la géomancie aussi bien tibétaine ou chinoise, qui cherche à ancrer le monastère dans un paysage protégé par des gardiens aux quatre points cardinaux ${ }^{29}$. Le paysage de chaque orient peut revêtir certaines formes, contenir certains éléments et en éviter d'autres, de mauvais augure, selon une cartographie idéale. Les prescriptions tibétaines renvoient à la cosmologie indo-bouddhique: le monastère est la montagne sumeru (un ma ṇdala ${ }^{30}$, une représentation cosmologique ${ }^{31}$ ), le paysage qui l'entoure représente l'univers, c'est pourquoi les traits qui figurent les quatre continents doivent être retrouvés aux points cardinaux. Certaines exigences de la géomancie peuvent ainsi expliquer des choix d'orientation autres que le sud.

L'ensemble de ces préceptes est à l'évidence impossible à rassembler en un seul lieu, aussi s'efforce-t-on de regrouper au mieux ces conditions. Par exemple, la grande chaîne des monts Qarayuna ou Daqingshan (au nord et à l'ouest de Kökeqota en Mongolie-Intérieure), orientée est-ouest, et faisant face, au sud, au fleuve Jaune, présente plusieurs conditions favorables, et de nombreux monastères ont choisi de s'y «adosser » (le Maidari-jin zuu, les monastères d'Üsütü près de Kökeqota, le Mergen süm-e des Urad, etc.).

D'après quelques récits de fondation qui nous renseignent sur l'application de ces préceptes, il était courant qu'un lama fondateur ou un géomancien parcourût pendant des mois, voire une année,un territoire en cherchant un signe faste qui lui indiquerait le site idéal. Les signes semblaient souvent être plus importants que les préceptes des traités de géomancie. Certains emplacements furent abandonnés à cause d'un mauvais présage $^{32}$. Une montagne qui ressemblait à une cloche de métal renversée (comme au monastère de Šongqur) ou bien un cirque de montagnes comparé à un lotus à huit pétales ou à la roue de la loi (comme au Zegün yar zuu de la bannière Zegün yar, ligue des Ordos, ou comme au Barayun keid, ligue d'Alašan) étaient en revanche d'excellents présages. Quand on est sur place, ces signes ne sont pas toujours évidents pour des yeux non avertis.

Des signes potentiellement néfastes peuvent être compensés et neutralisés par des actions bénéfiques antagonistes, comme la construction d'un temple ou d'un stūpa "géomantique" (pratique courante dans le monde bouddhique). Au monastère de Šongqur, on construisit un stūpa sur un îlot afin de protéger le monastère en expulsant les créatures malfaisantes qui peuplaient le lac. La divinité du solétant capable de provoquer des inondations et ainsi de renverser la pagode, on entoura pour plus de précaution le site de treize obos (Erihen 1986, p. 79).

Des problèmes de pollution liés à la géomancie peuvent survenir après la fondation. Par exemple, le monastère de Buryasutai (dans la bannière Otuy, ligue des Ordos) semblait être poursuivi par le mauvais sort. Les bâtiments furent détruits à deux reprises, et les moines disparurent les uns après les autres. Les géomanciens prescrivirent de reconstruire le monastère une troisième fois, plus à l'ouest (Van Hecken 1963, p. 136). De même, l'Ata-jin süm-e, dans la même bannière, fut démoli en 1950 et reconstruit sur un site nouveau, car son emplacement n'était pas en accord avec la position voulue par la géomancie (ibid.). Le monastère du Kambu blam-a (près d'Ouliastaï, province de Zavkhan, république de Mongolie) fut également "déménagé ", car son site fut reconnu comme néfaste et responsable de la maladie du Kambu blam-a (Pozdneev [1887] 1978, pp. 621-622 note 11). L'instabilité d'un site peut avoir de nombreuses causes liées à l'astrologie, à la 
colère de divinités qu'on a omis d'apaiser et à "l'épuisement énergétique»; le déménagement ou la réorientation est alors la seule solution.

\section{Plans d'ensemble des monastères mongols}

Les monastères préservés jusqu'au XXI ${ }^{e}$ siècle ont traversé des périodes troublées, en particulier les violentes persécutions religieuses de la fin des années 1930 en république populaire de Mongolie et la révolution culturelle chinoise en MongolieIntérieure. Dans la grande majorité des cas, les bâtiments préservés sont le temple principal - la grande salle d'assemblée où se réunissait la communauté monastique (youl coycin) et le sanctuaire abritant les principales statues du culte - et des temples de collèges monastiques ou des résidences de lamas réincarnés (qubilyan). Les sang (terme désignant à la fois les greniers et les trésors et l'administration des ressources et dépenses de chaque temple et de chaque lama réincarné), les habitations des moines, les cuisines, les imprimeries, les latrines, les écuries et autres bâtiments secondaires ont été rasés.

Des peintures et des photographies datant de la fin du XIX ${ }^{e}$ siècle et du début du $\mathrm{XX}^{\mathrm{e}}$ siècle nous informent sur l'aspect et l'étendue des plus grands monastères mongols $^{33}$. La hiérarchie entre les édifices est marquée par leur emplacement, ainsi que par leur hauteur, leurs dimensions, le nombre d'étages et leur ornementation. On peut constater, en règle générale, pour un monastère orienté vers le sud, que les temples principaux, points de convergence de la communauté, sont situés au centre du monastère et que les maisons des moines forment une ville au sud, sud-est et sud-ouest de ce noyau. Devant (au sud), une grande place est réservée aux danses rituelles cam. La cuisine est contiguë à la salle d'assemblée pour servir le thé pendant les offices. Comme au Tibet, l'emplacement des stūpa ne semble pas obéir à des règles particulières. Les sang et les résidences (labrang) des lamas réincarnés et des moines de haut rang se trouvent au nord, au nord-est ou au nord-ouest des temples. L'« arrière » (le nord) est plus honorifique que l'entrée: dans le bouddhisme tibétain, la tradition accorde au maître spirituel une place prééminente ; sa résidence sera donc plus « haut ", c'est-àdire au nord du temple. D'autre part, le côté droit (correspondant à l'ouest) est plus valorisé que le gauche : la principale réincarnation du monastère aura sa résidence à l'ouest, tandis que la réincarnation secondaire se contentera d'une résidence à gauche, à l'est. L'importance accordée au fond par rapport à l'entrée (que l'on retrouve dans l'architecture chinoise et tibétaine), et à la droite par rapport à la gauche ${ }^{34}$, rappelle l'organisation intérieure de la yourte mongole (ger) ${ }^{35}$. Les yourtes ont d'ailleurs abrité les premiers monastères de Mongolie Qalq-a sans que leur disposition intérieure en soit profondément modifiée : autel et statues au fond, banc pour les moines de part et d'autre.

31 L'observation du plan des monastères mongols permet de mettre en évidence l'adoption de conceptions chinoise, tibétaine et autochtone de l'espace. Une typologie par styles architecturaux généralement acceptée divise le corpus des monastères mongols en " plans chinois ", " plans tibétains » et «plans en forme de camp » hérités des campements mongols ${ }^{36}$. Cette classification simplificatrice s'applique rarement au plan d'ensemble des monastères. Les monastères dont nous connaissons l'étendue à la fin $\mathrm{du} \mathrm{XIX}^{\mathrm{e}}$ siècle ou au début du XXe siècle adoptaient fréquemment un plan global circulaire ("en forme de camp»), mais chaque complexe de temples, au centre, 
adoptait un plan « à la chinoise » dans une enceinte, ou un plan « tibétain ». Le meilleur exemple en est le monastère impérial Amurbajasqulangtu keid, dont la partie centrale des temples adopte un plan chinois alors que la forme d'ensemble était circulaire (« en forme de camp ») (fig. 2) - ce qui ne se voit plus aujourd'hui, les bâtiments résidentiels ayant été rasés. De nombreux monastères optent pour des enceintes individuelles à chaque temple ou complexe de temples, mêlant bâtiments chinois et tibétains (Erdeni $z u u$, Mergen süm-e, etc.) (fig. 3). La grande diversité des plans peut s'expliquer ainsi : chaque monastère a une histoire particulière, s'est agrandi progressivement ou a été d'emblée conçu sur une grande échelle. La multiplication des sanctuaires et des salles d'assemblée au cours du temps brouille la lisibilité du plan et peut modifier complètement un schéma initial ordonné et symétrique. Les nombreuses variantes respectent toutefois grosso modo les mêmes principes d'orientation.

Fig. 2. - Plan ancien d'Amurbajasqulangtu keid (Maidar 1972, fig. 113)

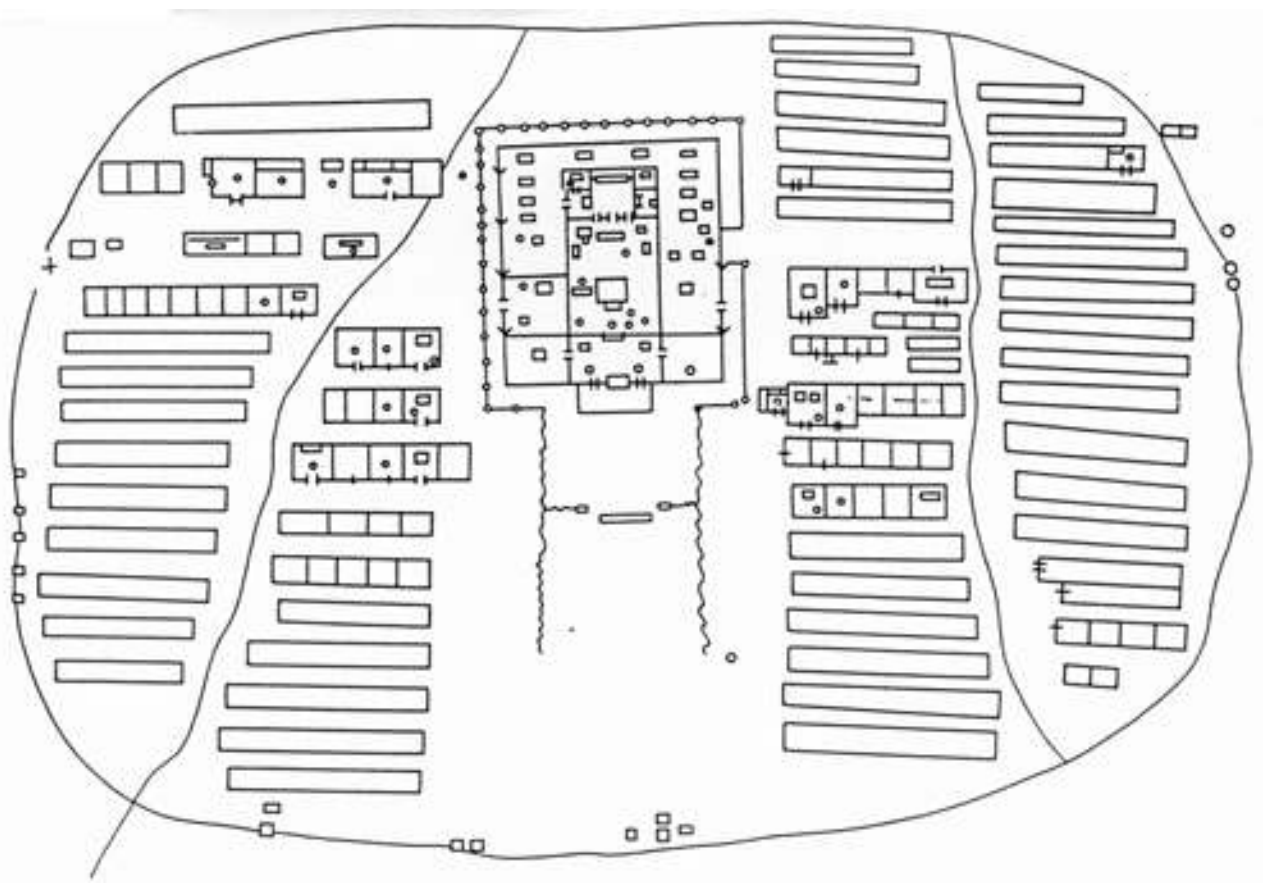


Fig. 3. - Plan du Zegün kürij-e sur la Kerülen, république de Mongolie (Maidar 1972, fig. 112).

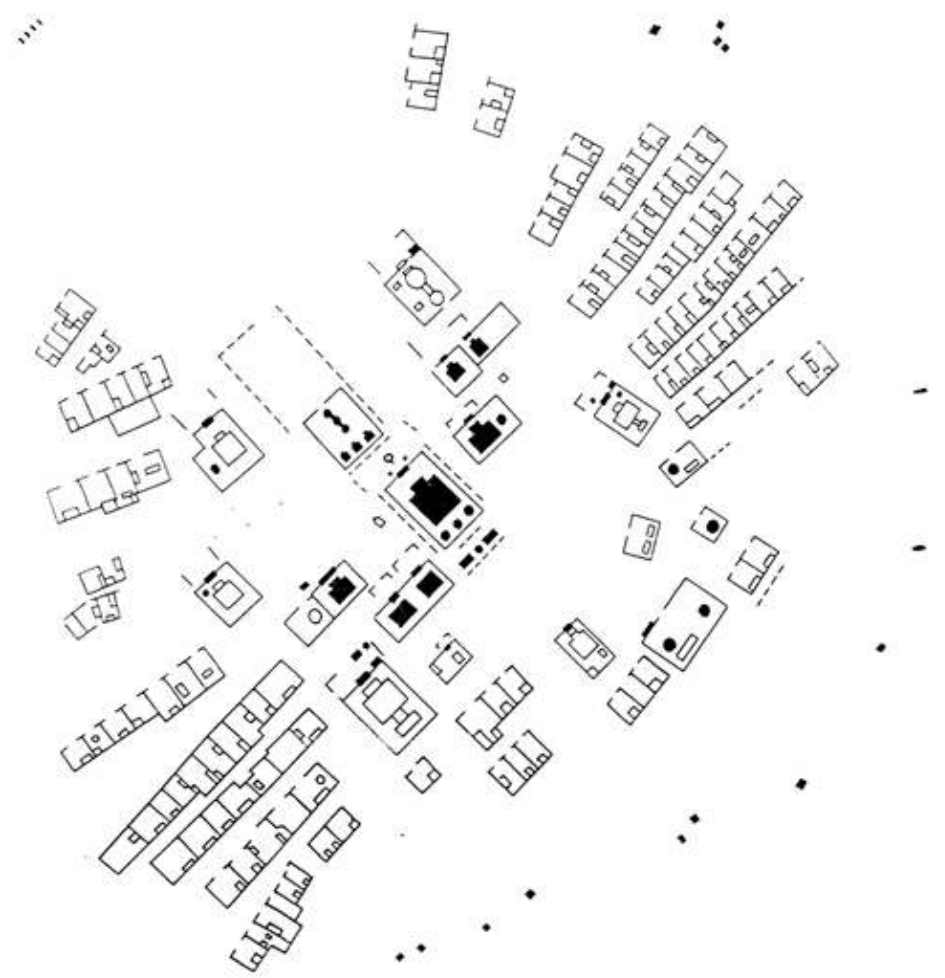

Les habitations des moines rayonnent autour du noyau central

32 Il est néanmoins permis de reprendre la typologie en plans « chinois », «tibétain » et « en forme de camp » en considérant qu'elle s'applique à des unités architecturales à l'intérieur d'un même monastère, plutôt qu'à l'ensemble du monastère.

Le plan d'origine chinoise, étiré le long d'axes orientés du sud au nord et entouré d'une enceinte rectangulaire, est fréquent en Mongolie-Intérieure, mais les dimensions, l'architecture et l'affectation des bâtiments sont adaptées au culte tibétain (fig. 4). 
Fig. 4. - Plan du Siregetü zuu de Kökeqota, Mongolie-Intérieure (Liu Dunzhen [1984] 1997, p. 381)

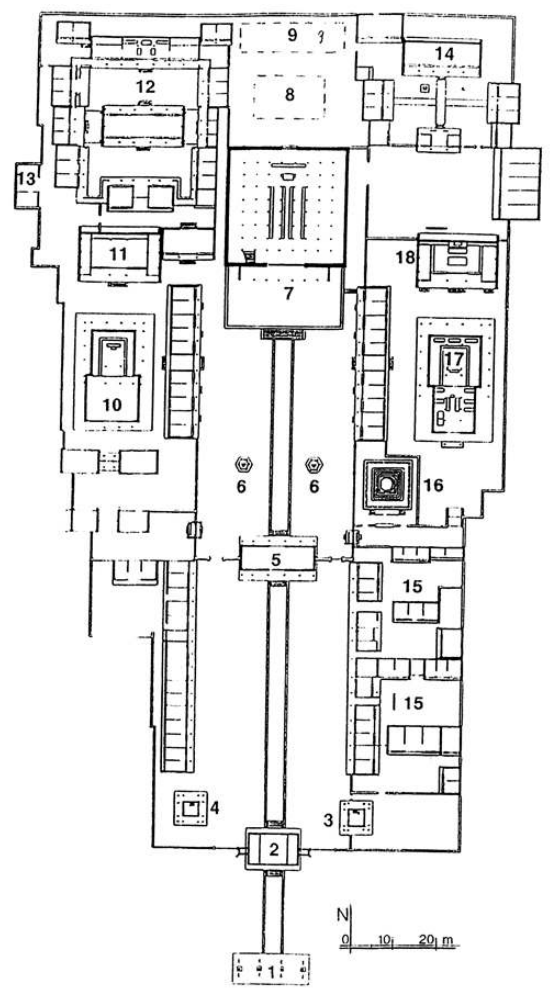

\footnotetext{
1. Portique triomphal (paifang)

2. Pavillon d'entrée

3. Pavillon du tambour

4. Pavillon de la cloche

5. Temple des arhat

6. Pavillons des stèles

7. Toul coycin

8. Sanctuaire principal [détruit]

9. Salle de neuf travées (Jiujian lou) [détruite]

10. Ancienne salle de bouddha (Gu fodian)

11. Temple du Kanjur (ou ancien temple : Gu miao)

13. Labrang du Siregetü qutuytu

14. Écuries

15. Labrang du Darqan corzi qutuytu

16. Habitations des moines

17. Stūpa

18. Temple de Nechung (ou temple de Pehar)

19. Temple du Tanjur
}

Une grande importance est donnée à la symétrie; de nombreux éléments vont par paire (mâts, lions, stūpa, pavillons identiques de la cloche et du tambour, salles latérales symétriques, etc.). En Chine, le monastère est comparé à une montagne: depuis la "porte de la montagne » (shanmen) qui perce le mur sud du temple, le dévot traverse des cours et des salles d'importance croissante dans sa progression vers les statues principales, au sommet de la "montagne ", i.e. vers le nord du temple. Toute visite à un temple est un pèlerinage à une montagne.La « hauteur » est alors représentée par l'axe horizontal sud-nord, les trois dimensions de l'espace étant en quelque sorte projetées en deux dimensions.

Le «plan tibétain » est, pour les auteurs mentionnés précédemment, un fourre-tout généralement qualifié d'« organique ", c'est-à-dire sans organisation apparente, sans ordre, parce que, situé en montagne, il suit les reliefs du terrain et que souvent la 
planification d'origine a été brouillée par des additions ultérieures (fig. 5). Dans le système de valeurs tibétain, le paysage est perçu comme étagé selon une hiérarchie qui valorise la hauteur ${ }^{37}$, mais le plan centré est également prisé. Hauteur et centre se rejoignent dans la cosmologie (mont Sumeru au centre de l'univers, palais-maṇdala, etc.), mais ne s'accordent pas dans le cas de monastères étalés sur la pente d'une montagne. Le temple principal (en tibétain gtsug lag khang) doit idéalement être à la fois au centre du monastère et plus " élevé » que les autres : on le trouve tantôt au nord du monastère (en haut de la pente), tantôt au centre (plus haut que les autres, ou surélevé par une terrasse). Les salles d'assemblée des facultés sont souvent alignées côte à côte avec le temple principal. Seules les résidences des moines réincarnéspourront être placées plus « haut » que les temples, mais leurs dimensions sont plus modestes.

Fig. 5. - Plan de la partie centrale du Badyar coiling süm-e près de Buyutu (Mongolie-Intérieure) au début du XIX es. (Nagao [1947] 1991, p. 137 fig. 2)

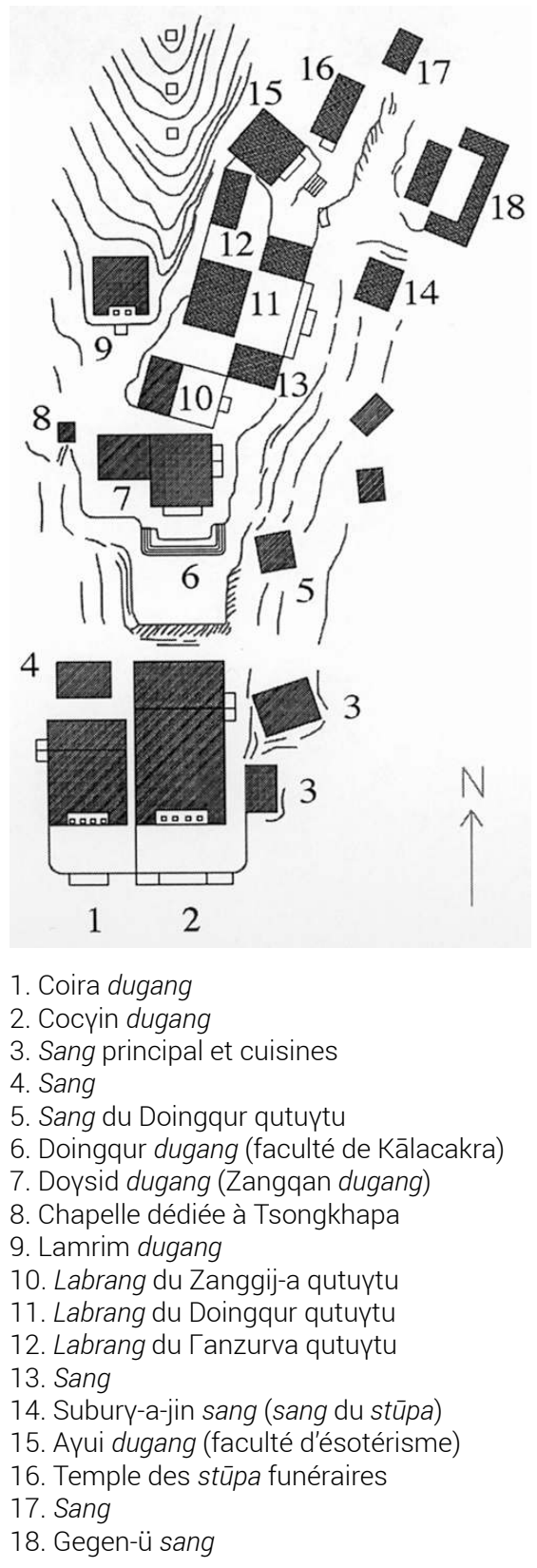

Études mongoles et sibériennes, centrasiatiques et tibétaines, 36-37| 2006 
Le plan en forme de camp mongol (ou d'enclos, d'anneau : kürij-e, terme qui en vint à signifier « monastère » $)^{38}$, caractéristique des monastères itinérants de Mongolie Qalq-a qui se fixèrent auX XVIII ${ }^{e}$ et $\mathrm{XIX}^{\mathrm{e}}$ siècles, place le grand temple au centre d'un cercle formé par les yourtes des moines. Ce plan centré faisait naturellement penser au maṇdala. Jeke Kürij-e (le Grand Monastère, qui se fixa vers 1788, appelé Ourga au $\mathrm{XIX}^{\mathrm{e}}$ siècle, puis Oulan-Bator) est décrit dans des chants bouddhiques et représenté en peinture comme unmaṇdala ${ }^{39}$ (fig. 6). Ce monastère géant était également comparé à la yourte, avec valorisation du nord par rapport au sud et de l'ouest par rapport à l'est, en raison de son organisation autour d'une vaste place centrale (correspondant à l'orifice zénithal de la yourte) et de la présence des districts riches au nord (Wasilewski 1976, p. 355).

Fig. 6 - Reproduction d'une peinture attribuée à l'artiste Zügder, exécutée vers 1912-1913, représentant Jeke Kürij-e et conservée au palais du Boyda gegen

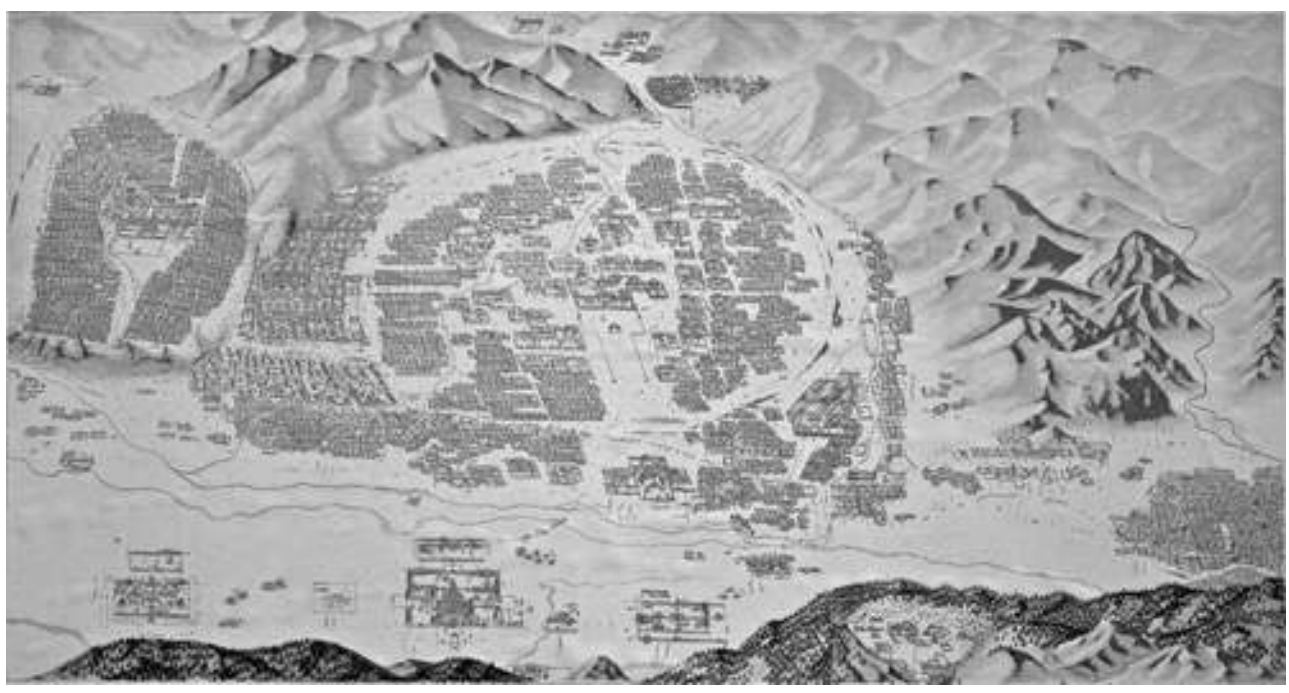

On y voit à l'ouest le monastère de Gangantegcilen

37 Certains monastères mongols ont poussé le souci défensif jusqu'à ériger trois enceintes imbriquées et concentriques : c'était le cas du temple de Cabcijal, fondé par les Tümed sur les berges du lac Koukounor, et du temple de Kouldja, le Yun bde lha khang, situé sur la rive nord de l'Ili. Ces temples, par leur plan, faisaient référence aumandala, mais il n'en subsiste aujourd'hui aucune trace.

Deux monastères fortifiés de Mongolie présentent un cas particulier, tant par la présence de leur muraille approximativement carrée que par leur organisation intérieure en partie préservée. Au Maidari-jin zuu, situé à l'ouest de Kökeqota et fondé fin $\mathrm{XVI}^{\mathrm{e}}$ début XVII siècle, les bâtiments centraux sont alignés sur un axe qui n'est parallèle ou perpendiculaire à aucun des murs de l'enceinte et ouvrent vers le sud-sudouest (fig. 7). 
Fig. 7 - Plan du Maidari-jin zuu (Jin Shen 1984, p.27)

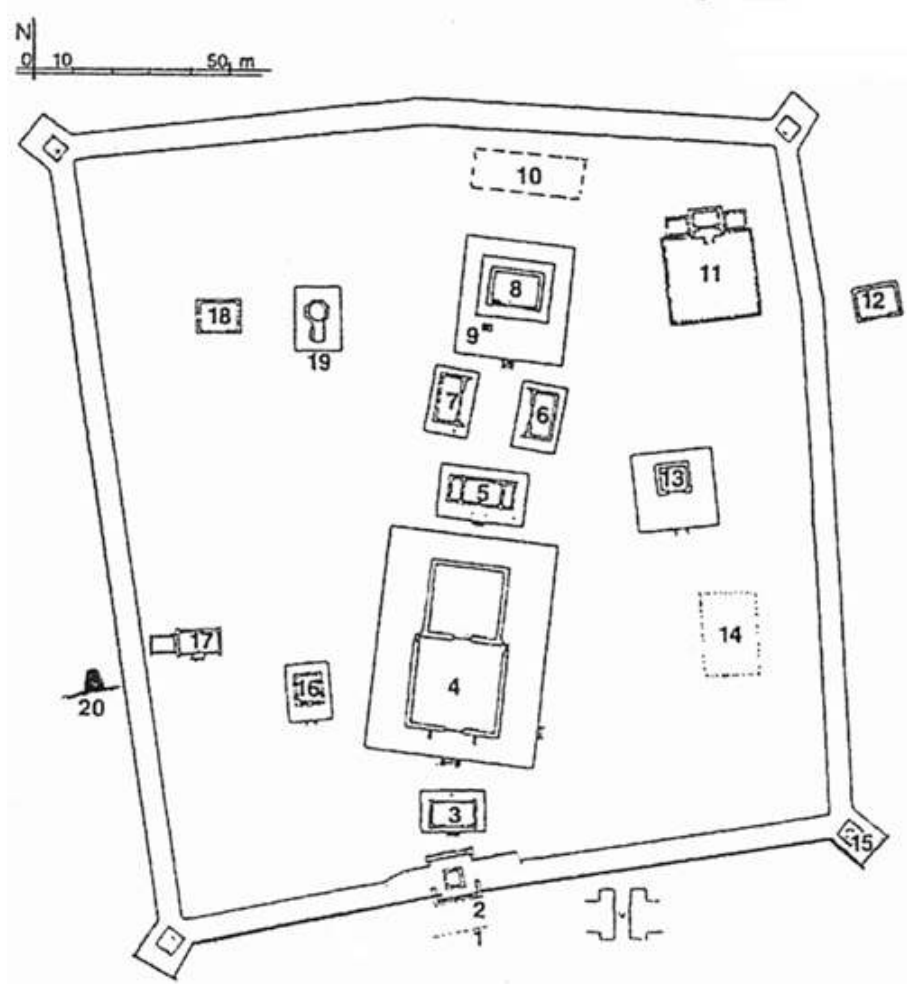

1. Mur-écran zhaobi [détruit]

2. Porte Taihe

3. Temple des lokapāla [détruit]

4. Гoul coycin

5. Temple du dieu des richesses [en ruine]

6. Temple d'Avalokiteśvara

7. Temple des 18 Arhat

8. Liuli dian (salle aux tuiles vernissées vertes)

9. Stūpa [détruit]

10. Gongye fu (Wangye fu)

11. Temple du dalaï lama

12. Temple oriental des 10000 bouddhas

13. Taihou miao (temple de l'impératrice)

14. Dajiwa dian [détruit]

15. Tours d'angle

16. Temple de Nechung (ou temple de Pehar)

17. Résidence du qutuytu

18. Temple occidental des 10000 bouddhas

19. Temple octogonal (Laojun miao)

20. Coupe du mur

Trois édifices sont orientés approximativement vers le sud, et cinq autres adoptent une orientation intermédiaire entre le sud et le sud-sud-ouest, plus ou moins parallèle aux murs est et ouest de l'enceinte (Charleux 1998, pp.68-71 et 316-344). À Erdeni zuu, célèbre monastère jouxtant les ruines de Qaraqorum en Mongolie Qalq-a, les premiers temples construits (trois temples d'Abatai Qan, 1586; temple du dalaï lama, 1675) ouvrent vers l'est-nord-est, mais l'enceinte plus tardive, érigée en 1743, et les bâtiments postérieurs adoptent un axe légèrement différent (fig. 8). 


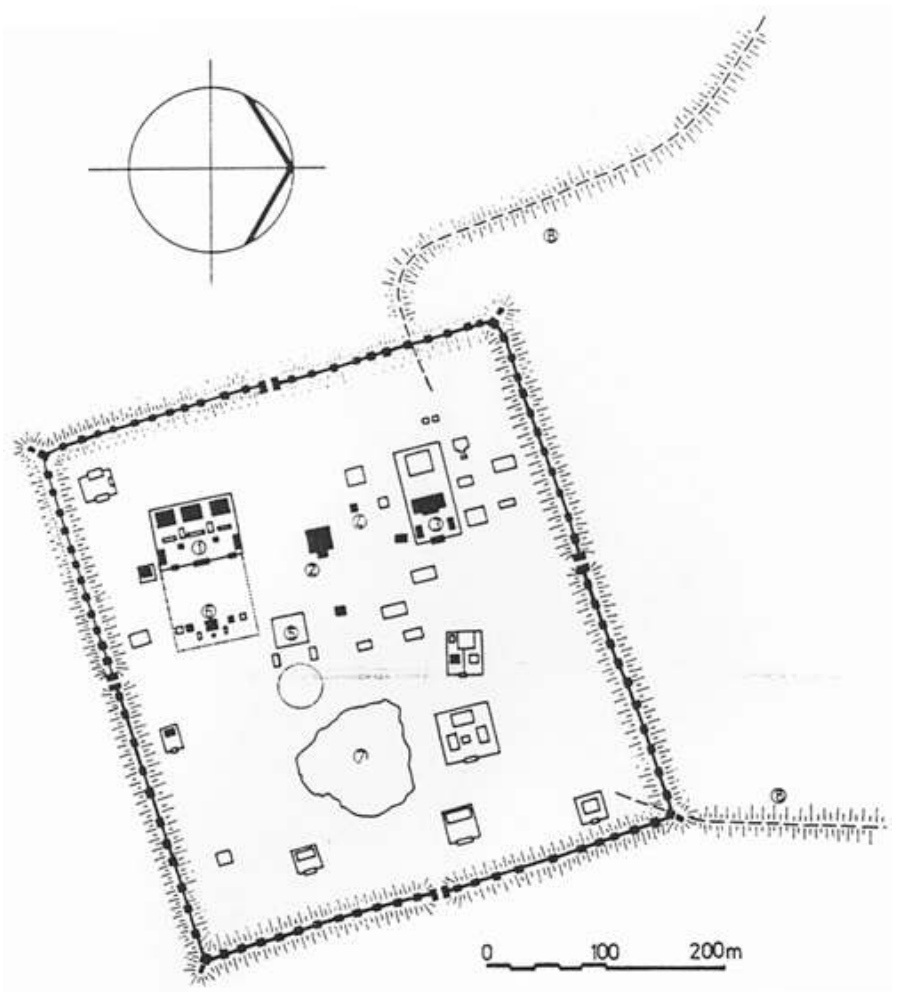

Dans les deux cas, les raisons de ces différentes orientations sont inconnues. Peut-être existe-t-il une explication géomantique (orientation jugée néfaste); il est également courant dans tout le monde bouddhique de modifier une orientation parce qu'un calcul initial est jugé faux : en tous cas la question de l'orientation semble avoir préoccupé les constructeurs. On remarque également qu'au Maidari-jin zuu comme à Erdeni zuu, aucun édifice remarquable n'occupe le centre de l'enceinte. Cette absence de marquage du centre, à l'opposé du modèle indien et du monastère-maṇdala, figure comme une exception en Mongolie ${ }^{40}$. Un célèbre dessin d'Erdeni zuu n'a pas tenu compte de ces orientations différentes et de l'absence de marquage du centre (fig. 9) : les architectures y sont bien alignées, l'enceinte est parfaitement carrée, et au centre se trouve la plateforme circulaire de la yourte de bois de vingt mètres de diamètre construite pour le Boyda gegen. Ce dessin est un exemple supplémentaire d'une représentation idéale éloignée de la réalité. 


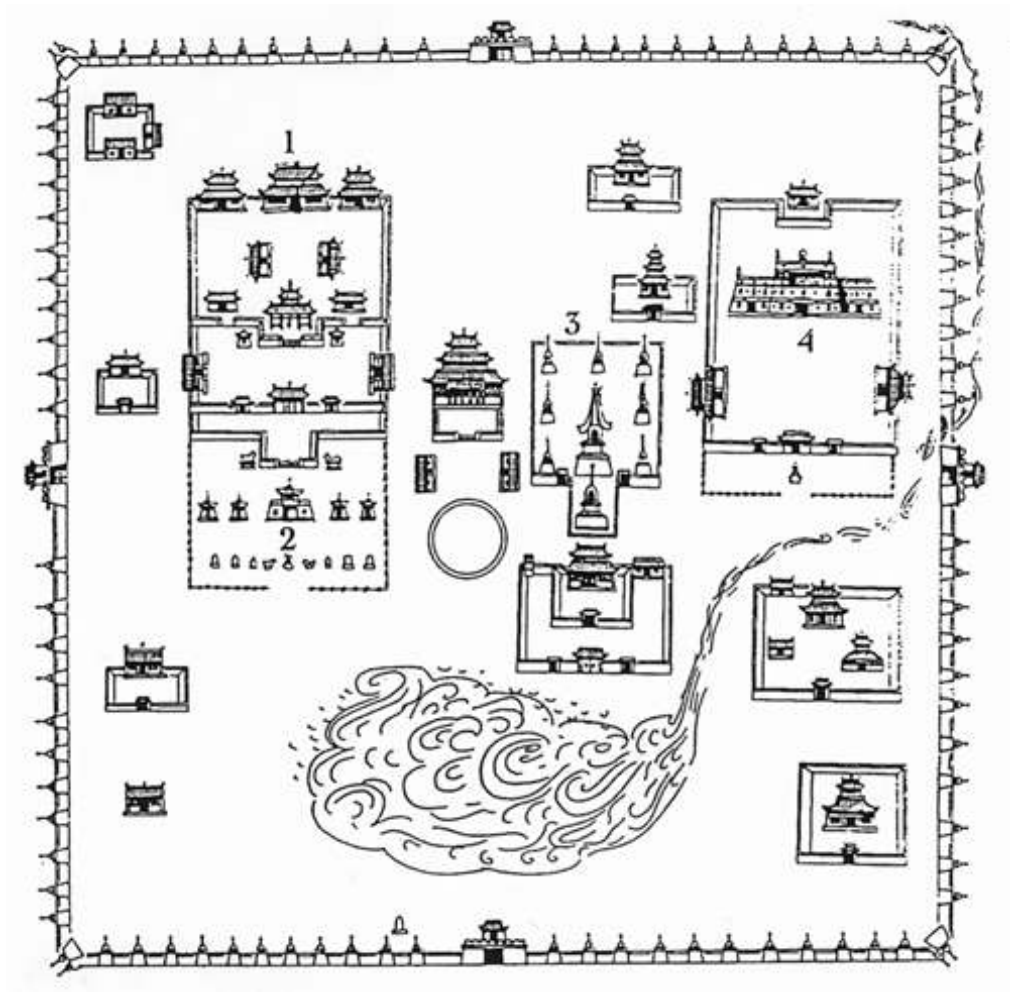

\section{Temples axiaux, temples-maṇdala et stūpa}

41 Au sein du monastère, les temples, constitués d'une salle d'assemblée à l'avant et d'un sanctuaire (qui peut encore être un bâtiment indépendant) à l'arrière, sont conçus pour être vus de face et n'ont souvent qu'une entrée. La façade du temple principal porte la décoration la plus riche, tandis que son mur nord ne comporte aucune ouverture ni décoration. C'est contre le mur nord que sont adossées, à l'intérieur, les principales statues tournées vers le sud, face aux fidèles.

La plupart des salles d'assemblée adoptent l'éclairage zénithal par un lanterneau ou puits de lumière, pour des raisons pratiques (la salle est grande et le rez-de-chaussée est souvent dépourvu de fenêtres) et symboliques. Le symbolisme tibétain du lanterneau met l'accent sur la lumière et la communication avec le ciel, tandis que le symbolisme mongol de l'orifice supérieur de la yourte (erüke), qui a pour fonction essentielle d'évacuer la fumée, se réfère au foyer ${ }^{41}$. Le terme chinois moderne tianjing (« puits du ciel »), de même que les termes tibétains composés de mthongs (« puits»), désigne à la fois le lanterneau et la cour intérieure de la maison et du temple ${ }^{42}$. Il faut donc voir dans le lanterneau, comme dans les courettes fermées, à la fois le symbolisme dumandala et l'idée de libre communication de la terre avec le ciel par un puits central.

Sous l'influence de l'architecture chinoise, qui traite salle d'assemblée et sanctuaire comme deux bâtiments distincts, et du modèle dumandala, certaines salles d'assemblée mongoles adoptent un plan centré autour d'un lanterneau ${ }^{43}$. Quand la salle comporte un étage, il est formé de quatre galeries entourant un couloir à ciel ouvert, lui-même entourant un lanterneau. La salle est alors quasiment cubique (si l'on excepte le porche) et son plan à l'étage rappelle les trois enceintes d'unmandala. Cette élévation 
est caractéristique des fondations impériales des Qing - comme la grande salle d'assemblée du monastère Amurbajasqulangtu (cf. chayet et jest 1991, p. 77 figures 7 et 8) et celledu Sir-asüm-e de Dolonnor -, mais se retrouve dans de nombreux autres monastères. Les Chinois ont coutume d'appeler ce type de temple «en forme de caractère hui », " sur le modèle du Potala de Lhasa ». Cette architecture n'est cependant pas d'origine chinoise (à l'époque Qing en tous cas ${ }^{44}$ ) ; elle fut reprise par les Qing dans des monastères tibétains de Chine qui imitent explicitement le Potala, comme le Xihuang si (temple Jaune de l'Ouest) à Pékin et le Putuozongcheng miao à Chengde.

$\mathrm{Au}$ contraire, le modèle tibétain du grand temple gelugpa (le gtsug lag khang), largement adopté en Mongolie dès le XVI ${ }^{\mathrm{e}}$ siècle (souvent avec des matériaux, des techniques et des couvertures chinois), réunit salle d'assemblée et sanctuaire en un seul bâtiment. Son profil en escalier, qui met l'accent sur le sanctuaire du fond, son iconographie et le cheminement du dévot qui doit le traverser du sud au nord en font un bâtiment axé. À l'intérieur, c'est donc le nord - le sanctuaire et ses statues - qui est mis en valeur (fig. 10).

Fig. 10 - Vue plongeante d'une salle d'assemblée typique des monastères de kökeqota (d'après Zhang Yubuan 1986, p. 341 fig. 10.3.7a)

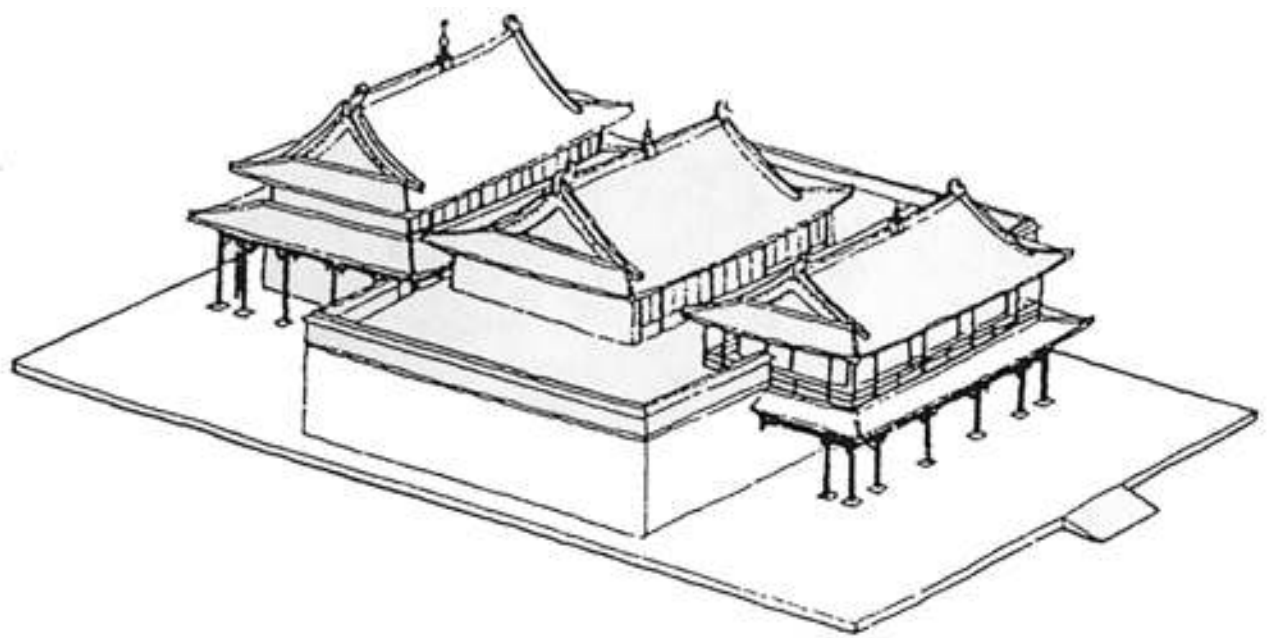

Cette concurrence entre le centre et le nord est bien connue des spécialistes de l'architecture bouddhique en général, et des historiens de l'architecture tibétaine en particulier, puisque d'anciens temples tibétains, à l'imitation de modèles indiens, adoptaient un plan centré en maṇdala (le Jo khang de Lhasa, le dBu rtse du monastère de Samye, le temple du monastère de Tho ling, etc.). Sous l'influence de l'habitat et de la conception de l'espace tibétains, l'évolution du grand temple tibétain après le $\mathrm{XV}^{\mathrm{e}}$ siècle a privilégié un plan axial.

L'architecture bouddhique mongole trouve son originalité dans la prédilection pour une organisation spatiale de type centrée - qui n'était déjà plus en vogue dans l'architecture tibétaine contemporaine -, unie à un goût pour des temples axés à volumétrie complexe. Cependant, même dans le modèle du temple-mandala, l'aménagement intérieur suit un axe sud-nord: les bancs des moines sont de part et d'autre d'une allée centrale, les trônes des réincarnations à droite et à gauche de l'allée, la table d'offrandes, les icônes et les textes religieux au fond, enfin l'iconographie (peinture murale et sculpture) suit une progression dans le panthéon, depuis les rois gardiens des quatre orients et les divinités protectrices courroucées au sud, les arhat ou 
les scènes narratives le long des parois latérales symétriques, les bodhisattvas, et enfin les bouddhas et les portraits de grands maîtres au nord.

Comme dans l'ensemble du monde bouddhique, le stūpa est la représentation cosmologique axée, centrée et orientée par excellence (voir Snodgrass 1985). Un stūpa mongol du XVIII e siècle, le Tabun subury-a (« cinq stūpa ») de Kökeqota, est, par son iconographie, sa forme (il est surmonté de cinq pagodons) et sa décoration, un maṇdala des bouddhas des cinq orients (les Jina). Le monastère dans lequel il était inséré était également dédié aux Jina, dont le culte était déjà important sous la dynastie Yuan (le maṇdala des cinq Jina est représenté sur la "plate-forme des nuages » de Juyong guan, bâtie en 1342-1345 au nord-ouest de Pékin) et dans la Chine des Ming.

On remarque en Mongolie un rapprochement explicite entre le stūpa et l'obo, autel des esprits locaux. Les manuels de construction des obos insistent sur leur poteau axial; autour, on doit retrouver les couleurs des quatre points cardinaux et les animaux gardiens (Gerasimova 1981, p. 167). Les obos construits par groupes de 13 (ou de 108) sont sous la plume des religieux syncrétistes une représentation du cosmos, au centre duquel s'élève le mont Sumeru. Stūpa et obos sont d'ailleurs souvent associés : on trouve côte à côte en haut de la colline dominant un monastère des obos entourant un stūpa, ou des stūpa entourant un obo.

\section{Orientation et circumambulation}

Dans le bouddhisme, l'orientation implique une dynamique, car la circumambulation dans le sens de la marche du soleil (pradakșinā; mongol : süm-e ergikü), héritée de l'hindouisme, est une pratique cultuelle essentielle ${ }^{45}$. L'acte de tourner autour d'un site sacré évoque l'enseignement de Bouddha qui fait tourner la roue de la $\mathrm{Loi}^{46}$. Qu'ils aient été influencés par le bouddhisme ou antérieurs à l'introduction du bouddhisme en Mongolie - ils sont en effet attestés dans la religion prébouddhique des Mongols ${ }^{47}-$, la circumambulation et les mouvements giratoires sont fréquents dans les rituels et dans les actions quotidiennes des Mongols : autour des tentes, des arbres et des obos, pour honorer un chef, célébrer un sacrifice, appeler la bonne fortune et, de manière plus générale, symboliser la circulation de la force vitale entre le monde des hommes et la surnature ou le monde des ancêtres ${ }^{48}$.

Dans le bouddhisme mongol, les processions du char de Maitreya (mongol: Maidari), organisées généralement le dernier jour des fêtes du nouvel an, témoignent de l'importance de la pratique de la circumambulation. La procession faisait des haltes aux quatre points cardinaux pour chanter "les Cinq Traités de Maitreya». La plus spectaculaire se tenait à Jeke Kürij-e (Ourga) et faisait le tour de la cité monastique. De même, à Kökeqota, les processions rituelles faisaient le tour de l'ensemble de la ville et de ses temples. Au Maidari-jin zuu, le char de Maitreya était hissé sur la muraille pour en faire le tour. La circumambulation se pratiquait également à l'échelle de la bannière: en cas d'épidémie ou de catastrophe naturelle, les moines des différents monastères s'unissaient pour effectuer le tour de leur bannière en une procession de chameaux portant les volumes du Kanjur ${ }^{49}$. Aujourd'hui, les pèlerins font encore le tour des monastères, en portant parfois des textes religieux sur le dos en vue d'une guérison (pratique observée au Sir-a mören süm-e en 1995). 
51 La forme de circumambulation individuelle principale consiste à contourner les stūpa et les statues du sanctuaire, à faire le tour du lanterneau à l'étage de la salle d'assemblée et à tourner autour de l'ensemble du monastère, le long d'un chemin prévu à cet effet où peuvent être disposés des moulins à prières. Un des termes génériques tibétains pour " pèlerinage ", gnas skor, signifie littéralement " tourner autour d'un site sacré ». Aujourd'hui, on peut voir les pèlerins faire la circumambulation autour du monastère d'Erdeni zuu, à l'extérieur des murailles.

52 Alors qu'au Tibet un espace étroit est souvent ménagé entre les statues cultuelles et le mur du fond du sanctuaire, dans les monastères de Mongolie, les statues sont placées contre le mur. Peut-être n'en a-t-il pas toujours été le cas: la finition des dos des statues du célèbre Zanabazar (le I ${ }^{\text {er }}$ Zibcündamba qutuүtu, également connu sous le nom d'Öndür gegen), par exemple, permet de penser qu'elles ont été conçues pour être également vues de dos. Aujourd'hui, les pèlerins pratiquent la circumambulation des statues à l'extérieur du sanctuaire, et de nombreux temples sont entourés sur leurs trois côtés de moulins à prières protégés par un auvent extérieur sur lesquels les dévots collent des petits papiers formulant des vœux ( $c f$. les temples du monastère de Gandan à Oulan-Bator). La circumambulation extérieure des statues a parfois entraîné certains aménagements architecturaux, telle la construction d'un couloir aveugle autour du sanctuaire ( $c f$. les trois temples d'Abatai Qan à Erdeni zuu) ou le percement de deux portes dans le mur nord de la salle d'assemblée ( $c f$. les temples de Kökeqota) ${ }^{50}$. Ces aménagements furent progressivement abandonnés au XVIII ${ }^{\mathrm{e}}$ siècle, et ces portes nord furent bouchées. Ouvrir une salle d'assemblée au nord représentait manifestement un danger géomantique. Une ou deux portes au nord pouvaient être nécessaires pour passer dans la cour suivante ou pour d'autres raisons ${ }^{51}$, mais l'on s'abstenait de percer un mur nord si l'on pouvait l'éviter.

53 La circumambulation fait du parcours du fidèle dans le temple un parcours tant spatial que temporel. Toutefois le programme iconographique obéit plus à des soucis de progression vers l'autel (du sud au nord) et de symétrie gauche/droite, et n'est pas déterminé par le parcours de circumambulation, comme il l'est dans le temple hindou.

\section{Conclusion}

54 Plus encore que les Mongols, les Mandchous ont cherché à construire des villes et des monastères centrés et orientés. Moukden, leur ancienne capitale, est représentée comme un maṇdalaorienté avec ses quatre monastères aux quatre orients (cf. les temples construits par Hong taiji de 1643 à 1645). Le Pékin mandchou a été comparé à un maṇdala de Vajrabhairava avec en son cœur le stūpa blanc construit sur l'île Qionghua du lac Beihai. L'empereur Qianlong, assisté du Zanggij-a qutuүtu Rol pa'i rdo rje, fit édifier plusieurs temples sur le modèle d'unmaṇdala, comme le Yuhua ge dans la Cité Interdite (restauré en 1750), maṇdala en trois dimensions sur quatre étages suivant le système des kriyā, carya $\bar{a}$, yoga et anuttara yogatantra, ou encore le Pule si de Chengde conçu comme un maṇdala de Cakrasampvara (1766-1767), le temple étant aligné avec le pic sur un plan géomantique couvrant l'ensemble de la vallée (Stoddard 1999, pp. 210-212).

Les Mongols de la dynastie Yuan avaient déjà montré l'intérêt qu'ils portaient à des modèles cosmologiques centrés et orientés pour l'architecture de leurs capitales ${ }^{52}$. Dans 
l'architecture bouddhique, les Mongols interprétèrent les modèles tibétains et chinois en fonction de leur vision du monde. Ils adoptèrent l'image centrée du cercle (mandala), en accord avec leur propre conception de l'espace (yourte, disposition circulaire des campements, représentation des cartes géographiques) et la développèrent dans certaines formes architecturales, sans doute sous l'influence de modèles venus d'Amdo et de Chine: temples carrés à galeries imbriquées, temples surmontés d'étages en réduction, temples entourés d'enceintes imbriquées. L'architecture accorde dans l'esthétique une grande importance à la symétrie, liée symboliquement à ce qui est droit et faste. Toutefois, le plan d'ensemble des monastères mongols est rarement ordonné, devant s'adapter aux caractéristiques du terrain et évoluant selon les agrandissements.

Lorsque l'architecture ne traduit pas la vision mandalique du monde, celle-ci est transposée dans le discours ou la représentation picturale : monastère vu comme une montagne, un mandala ou une yourte ; temple situé en plaine, décrit comme situé sur une forte pente; plan d'un monastère organisé selon une symétrie parfaite. Si les prescriptions nombreuses et complexes ne permettent pas de trouver le site idéal, des signes de bon augure, la construction d'un stūpa ou l'appellation de "montagne » donnée à une petite élévation compenseront ou corrigeront des défauts géomantiques. Les règles sont interprétées par des spécialistes qui savent reconnaitre dans le paysage un lotus à huit pétales ou la forme des dix-huit arhat. Il importe (pour nous) de distinguer le discours de la réalité et de comparer les sources avec la nature concrète du terrain.

57 L'architecture bouddhique révèle ainsi les tensions entre les considérations d'ordre pratique, spirituel, symbolique et artistique, entre les influences et sources d'inspiration diverses et les pratiques locales, entre une conception linéaire et axiale de l'organisation du temple chinois et l'attrait pour l'architecture cosmologique. La hiérarchie sud/nord est en tension avec la hiérarchie centre/périphérie tant dans l'organisation spatiale générale du monastère que dans chacun des coycin. Ces conflits sont résolus différemment, en jouant sur l'emplacement, l'altitude, la hauteur du bâtiment et/ou la hauteur de ses toits, en effectuant des compromis entre les traditions religieuses tibétaines et les traditions architecturales chinoises, pour aboutir à des plans et à des bâtiments composites. De fait, il n'existe pas de monastère-type, ni de monastère se conformant de près à un modèle explicite.

Le symbolisme microcosmique de l'organisation de la yourte, "véhicule identitaire " des Mongols, a fait l'objet de nombreuses publications ${ }^{53}$. Il a été montré à quel point la yourte est un indicateur des changements politiques, sociaux et religieux, la représentation de son espace évoluant selon les époques et incorporant de nouvelles "idéologies»; le système de représentation prébouddhique des Mongols reste cependant trop mal connu pour que l'on puisse distinguer ce qui est attribuable aux influences chinoises, bouddhiques et autres qui se sont exercées à différentes périodes $^{54}$. La question de l'orientation des temples mongols pourrait certainement être enrichie par des études plus précises sur la conception de l'espace, l'importance des points cardinaux et de l'orientation exacte ainsi que sur la valeur accordée aux orients - études menées grâce à des enquêtes sur le terrain et à l'examen des données de la littérature, des proverbes en particulier ${ }^{55}$. 


\section{Glossaire des termes chinois et japonais}

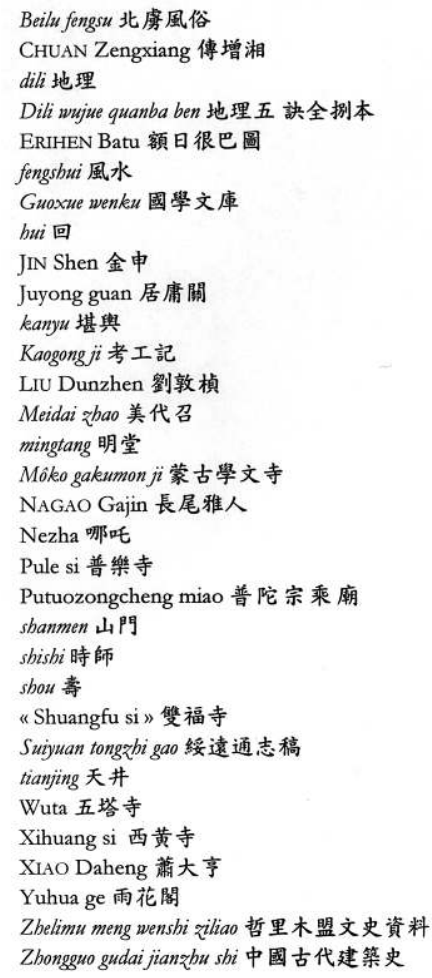

\section{BIBLIOGRAPHIE}

Aүula ba nutuү-un sinzi-jin bicig [Les caractéristiques des montagnes et des pâturages]

s. d. Manuscrit, Bibliothèque royale de Copenhague, Mong. 16.

Alexandre, E.

1993 L'architecture religieuse en pays khalkha, in Trésors de Mongolie. XVIIe-XIXe siècles (Paris, Réunion des Musées Nationaux), pp. 82-97.

Aris, Michael

1979 Bhutan: The Early History of a Himalayan Kingdom (Warminster, Aris \& Phillips).

Bara'nski, Marek

1991 Erdene-zuu Monastery. Conservator's remarks, in The Megzid Janraiseg Temple (Varsovie, The Ateliers for conservation of cultural property [PKZ] \& Foreign trade Office [BHZ]), pp. 42-49 [The Polish-Mongolian mission for conservation of historic monuments 1989, I].

Bawden, C. (éd.)

1961a The Jebtsundamba Khutukhtus of Urga (Wiesbaden, Otto Harrassowitz) [Asiatische Forschungen, 9]. 
Bawden, C.

$1961 \mathrm{~b}$ The supernatural element in sickness and death according to Mongol tradition, Asia Major New Series, VIII, 2, pp. 215-257.

Beffa, M.-L. et R. Hamayon

1983 Les catégories mongoles de l'espace, Études mongoles et sibériennes, 14, pp. 81-111.

Chabros, K.

1988 Space and movement in Mongolian culture, Journal of the Anglo-Mongolian Society, XI, 1, pp. 30-38.

Chabros, K. et S. Dulam

1990 La nomadisation mongole : techniques et symbolique (Bloomington, Indiana University) [Papers on Inner Asia, 13].

Charleux, I.

1998 Histoire et architecture des temples et monastères lamaïques de Mongolie méridionale. Thèse de doctorat de l'Université de Paris IV-Sorbonne.

Chayet, A.

1994 Art et archéologie du Tibet (Paris, Picard).

Chayet, A. et C. Jest

1991 Le Monastère de la Félicité Tranquille, fondation impériale en Mongolie, Arts Asiatiques, 46, pp. $72-81$.

Chuan, Zengxiang

1941 Suiyuan tongzhi gao [Monographie générale du Suiyuan] (Kökeqota, Nei Menggu daxue tushuguan).

Dili wujue quanba ben [Les cinq secrets de la géomancie]

s. d. Manuscrit, Bibliothèque royale de Copenhague, Mong. 107.

Ene anu yazar-un sinzin bolai [Voici les caractéristiques du terrain]

s. d. Manuscrit, Bibliothèque royale de Copenhague, Mong. 323.

Erihen, Batu

1986 Shuangfu si [Le monastère Shuangfu], Zhelimu meng wenshi ziliao (Tongliao), 2, pp. 76-87.

Even, M.-D.

1988-1989 Chants de chamanes mongols, numéro double spécial des Études mongoles et sibériennes, 19-20.

Even, M.-D. et R. Pop

1994 Histoire secrète des Mongols (Mongghol-un ni'uca tobciyan). Chronique mongole du XIIIe siècle (Paris, Gallimard).

Feuchtwang, S. D. R.

1974 An Anthropological Analysis of Chinese Geomancy (Vientiane, Vithagna).

Gaborieau, M.

1995 Villes de toiles et villes de pierre. Les capitales mogholes étaient-elles des camps ?, in P. Clément, S. Clément-Charpentier et C. Goldblum (éd.), Cités d'Asie, (Paris, Parenthèses), pp. 15-34.

Tazar-un sinzi-jin sudur [Traité des caractéristiques du terrain]

s. d. Manuscrit, Bibliothèque royale de Copenhague, Mong. 314. 
Gerasimova, K. M.

1981 De la signification du nombre 13 dans le culte des obo, Études mongoles et sibériennes, 12, pp. 163-175.

Haltod, M.

1966 Mongolische Ortsnamen, Teil I. Aus mongolischen Manuscript-Karten (Wiesbaden, Franz Steiner Verlag).

Hamayon, R.

1978 Des fards, des mœurs et des couleurs, in Serge Tornay (éd.), Voir et nommer les couleurs

(Nanterre, Laboratoire d'Ethnologie et de Sociologie comparative), pp. 206-247.

1990 La chasse à l'âme. Esquisse d'une théorie du chamanisme sibérien (Nanterre, Société d'ethnologie).

Heissig, W.,

1959 Die Familien und Kirchengeschichtsschreibung der Mongolen (Wiesbaden, Otto Harrassowitz), vol. I.

1978 Mongolische Ortsnamen, Teil II. Mongolische Manuscript-Karten in Faksimilia (Wiesbaden, Franz Steiner Verlag).

Heissig, W., assisté par C. Bawden

1971 Catalogue of Mongol Books, Manuscripts and Xylographs (Copenhague, The Royal Library).

Herdick, R.

1993 Remarks on the orientation of the large stūpas in the Kāṭmịānḍu Valley : A discussion of principles of lunar ordering, in C. Ramble et M. Brauen (éd.), Anthropology of the Himalaya (Zurich, Ethnological Museum of the University of Zurich), pp. 101-123.

Jagchid, S. et P. Hyer

1978 Mongolia's Culture and Society (Boulder, Colorado/Folkestone, G.-B., Westview Press/Dawson).

Jin, Shen

1984 Meidai zhao [Le Maidari-jin zuu] (Kökeqota, Nei Menggu renmin chubanshe).

Kotwicz, W.

1927 Sur les modes d'orientation en Asie centrale, Rocznik Orjentalistyczny (Lwów), 5, pp. 68-91.

Kürelša

1993 Zirim-ün süm-e keid [Les monastères du Zirim] (Hailar, öbür Mongyul-un sojul-un keblel-ün qorij-a) [Mongyul ündüsüten-ü süm-e keid-ün bürin ciүulүa, I].

Laudrin, F. et G. André

2003 Topographie et cartographie, in Mongolie, le premier empire des steppes (Arles/Paris, Actes Sud/Mission Archéologique Française en Mongolie), pp. 91-103.

Liu, Dunzhen,

[1984] 1997 Zhongguo gudai jianzhu shi [Histoire de l'architecture ancienne chinoise] (Beijing, Zhongguo jianzhu gongye chubanshe).

Maidar, D.

1972 Mongolyn arxitektur ba xot baiguulalt [Architecture et construction des villes en Mongolie] (OulanBator, Ulsyn xevlelijn gazar).

Miller, R. J.

1959 Monasteries and Culture Change in Inner Mongolia (Wiesbaden, Otto Harrassowitz) [Asiatische Forschungen, 2].

Meyer, F. et C. Jest

1987 Milieux, matériaux, et techniques, in P. Mortari Vergara et G. Béguin (éd.), Demeures des 
hommes, sanctuaires des dieux. Sources, développement et rayonnement de l'architecture tibétaine (Rome, Università di Roma « La Sapienza »), pp. 146-167.

Mostaert, A.

1956 Matériaux ethnographiques relatifs aux Mongols Ordos, Central Asiatic Journal, 2, pp. 241-294.

Nagao, Gajin

[1947] 1991 Mōko gakumon ji [Monastères académiques en Mongolie] (Kyoto, Chūkō bunko).

Pegg, C.

2001 Mongolian Music, Dance and Oral Narrative. Performing Diverse Identities (Seattle/Londres, University of Washington Press).

Pichard, P. et F. Lagirarde (éd.)

2003 The Buddhist Monastery. A Cross-cultural Survey (Paris, École Française d'Extrême-Orient).

Pozdneev, A. M.

[1887] 1978 Religion and Ritual in Society. Lamaist Buddhism in Late 19th in Mongolia (Bloomington, The Mongolia Society) [The Mongolia Society Occasional Papers, 10].

Pritsak, O.

1954 Orientierung und Farbsymbolik. Zu den Farbenbezeichnungen in den altaischen

Völkernamen, Saeculum, V, 4, pp. 376-383.

Ränk, G.

1949 Einige Bemerkungen über die traditionelle Solarorientierung bei den Nomadenbehausungen Asiens, Ethnos, XIV, 2-4, pp 150-159.

Rubrouck, G. de

1997 Voyage dans l'empire mongol, 1253-1255, trad. et commentaire de C.-C. Kappler et R. Kappler (Paris, Imprimerie Nationale).

Sarközi, A.

1976 A Mongolian manual of divination by means of characteristics of the land, in D. Sinor (éd.), Tractata Altaica (Wiesbaden, Otto Harrassowitz), pp. 583-604.

Sauer, C.

2001 The resurgence of tradition in a post-communist society. The role of the Mongolian 'ger' as a vehicle for the maintenance of ideology and practice in the diachronic process of Mongolian society, Central Asiatic Journal, XLV, 1, pp. 63-127.

Serruys, $\mathrm{H}$.

1945 Pei-lou fong-sou. Coutumes des esclaves septentrionaux de Siao Ta-heng, Monumenta Serica, X, pp. 117-208.

Smith, R. J.

1991 Fortune-Tellers and Philosophers. Divination in Traditional Chinese Society (Boulder, Colorado/San Francisco/Oxford, Westview Press).

Snodgrass, A.

1985 The Symbolism of the Stūpa (Ithaca, NY, Cornell University).

1990 Architecture : Time and Eternity. Studies in the stellar and temporal Symbolism of traditional Buildings (New Delhi, Aditya Prakashan), 2 vol. [Sata-Pi.taka Series, 356].

Stein, R. A.

1987 Le Monde en petit. Jardins en miniature et habitations dans la pensée religieuse d'Extrême-Orient (Paris, Flammarion). 
Stoddard, $\mathrm{H}$.

1999 Dynamic structures in Buddhist mandala : Apradaksina and mystic heat in the mother Tantra section of the Anuttarayoga tantras, Artibus Asiae, LVIII, 3-4, pp. 169-213.

ŠČepetil'nikov, N. M.

1960 Arxitektura Mongolii [L'architecture de la Mongolie] (Moscou, Gosudarstvennoe izdatel'stvo literaturi po stroitel'stvu).

Tangad, D.

1990 Coutumes mongoles liées au poteau de yourte, Études mongoles et sibériennes, 21, pp. 37-57.

Thubten Legshay Gyatsho

1980 Gateway to the Temple. Manual of Tibetan monastic Customs, Art, Building and Celebrations, trad. du tibétain par D. Jackson (Kāṭhmāṇḍu, Ratna Pustak Bhandar) [Bibliotheca Himalayica, 3].

Van Hecken, J.

1963 Les Lamaseries d'Otoy (Ordos), Monumenta Serica, XXII, 1, pp. 121-168.

Wasilewski, J.

1976 Space in nomadic cultures, in W. Heissig (éd.), Altaica Collecta. Berichte und Vorträge der XVII. PIAC (3.-8. Juni 1974, Bonn) (Wiesbaden, Otto Harrassowitz), pp. 345-360.

Xiao, Daheng

[1595] 1935 Beilu fengsu (Pékin, Wendiange) [Guoxue wenku, 29].

Zhang Yuhuan (éd.)

1986 History and Development of Chinese Architecture (Pékin, China Academy of Sciences).

\section{NOTES}

1. La «Mongolie » désigne dans cet article l'ensemble de l'aire culturelle mongole; la Mongolie actuelle sera appelée «république de Mongolie» ou «Mongolie Qalq-a » pour éviter toute confusion; la Mongolie-Intérieure (M.-I.) ou Mongolie méridionale est l'actuelle région autonome de Mongolie-Intérieure en Chine.

2. L'architecture urbaine $\mathrm{du} \mathrm{xx}^{\mathrm{e}}$ siècle, sur un modèle soviétique tant à Oulan-Bator qu'à Kökeqota ou dans les nouvelles métropoles de Mongolie-Intérieure, n'a pas tenu compte de cette orientation traditionnelle, ce qui déroute les éleveurs en visite à la ville.

3. À la fin $d u x^{2} I^{e}$ siècle, xiao Daheng, un observateur chinois des coutumes des Mongols Tümed et Ordos, remarque que les Mongols orientent la porte de leur tente vers le sud-est, ou vers le sud depuis qu'ils sont sous l'influence des moines tibétains (Beilu fengsu 1595, trad. par serruys 1945, pp. 143-144).

4. D'après Rubrouck (1997, p. 82), qui voyage dans l'empire mongol en 1254, les yourtes étaient orientées vers le sud, mais l'est était peut-être aussi une ancienne orientation de référence. Sur ce sujet, voir Kotwicz (1927, pp. 82-84) et Ränk (1949).

5. Les termes désignant les points cardinaux ne s'appliquent pas aux mêmes orients dans tous les dialectes mongols: barayun, "la droite», et zegün, "la gauche», désignent respectivement l'ouest et l'est chez les Qalq-a et les Caqar, le sud et le nord chez les Ordos (Beffa et Hamayon 1983 ; Kotwicz 1927, p. 90). Les Mongols ont adopté le système sino-turc à quatre points cardinaux (dörben züg), quatre directions intermédiaires (zobkis) plus le centre, ce qui donne le chiffre neuf, sacré dans le monde mongol (pritsak 1954).

6. La question du choix de la direction vers laquelle sont orientées les constructions dans les steppes de l'Asie intérieure est extrêmement problématique. Les différences d'orientations entre 
architectures palatiale, religieuse et funéraire et la difficulté de généraliser à partir de corpus de sites archéologiques souvent peu nombreux et mal datés ne permettent pas de donner des conclusions définitives (les Xiongnu privilégiaient le sud, l'est ou le nord ; les Ouïgours, l'est puis le sud, sans exclure l'ouest !, etc.). Voir par exemple Pritsak (1954, pp. 381-382), Kotwicz (1927, pp. 82-84) et Ränk (1949).

7. dGe lugs pa. [Dans le corps de l'article, les termes tibétains les plus connus ne sont pas écrits selon leur translittération, mais selon l'orthographe simplifiée en usage dans la littérature occidentale.]

8. La tête du Bouddha lors de son parinirvānaaétait orientée vers le nord, ce qui a été source de discussions dans la littérature bouddhique. En Inde, les premiers vihāra étaient tournés vers le sud, direction de la mort et des enfers dès l'époque védique. Par la suite, les monastères et les sanctuaires adoptèrent une direction plus faste, l'est (monastère de Nālandā, monastères des pays d'Asie du SudEst, sanctuaires bouddhiques d'Asie centrale).

9. $s K u$ 'bum.

10. A mdo.

11. On sait combien l'orientation est importante dans le monde indien, où de complexes calculs astrologiques déterminent le plan du temple-montagne hindou ou bouddhique (snodgrass 1985, pp. 15-36 et 1990, pp. 128-224 sur les méthodes de calcul des orients en référence au mouvement du soleil, notamment avec un pilier-gnomon). Une attention extrême consacrée à l'orientation des stūpa se retrouve dans les constructions bouddhiques de la vallée de Kathmandou (Herdick 1993). Aucune étude n'a été réalisée à ce sujet sur les temples tibétains et mongols.

12. Laudrin et André (2003, p. 101) font la même remarque au sujet des tombes xiongnu de Gol Mod en Mongolie: "Nous pensons plutôt que les axes de ces complexes funéraires ont été implantés non pas vers le nord, mais plutôt vers une idée de nord... ».

13. Soit la majorité des monastères de Mongolie-Intérieure. Les monastères des Qalq-a étaient généralement itinérants et se fixèrent à partir du XVIII ${ }^{\mathrm{e}}$ siècle.

14. Cf. par exemple les cartes dessinées entre 1890 et 1920 sur ordre impérial, conservées en Allemagne, dans la bibliothèque de Marbourg : Haltod (1966) et Heissig (1978).

15. Dans la cosmologie bouddhique issue de la tradition brahmanique, l'univers a la forme d'un disque traversé en son centre par une montagne, le mont Sumeru, identifié par les Tibétains au mont Kailaś (tibétain : Ti se). Cet axe du monde, résidence des dieux, est entouré de sept océans et de quatre continents situés à chacun des orients, le continent du sud, Jambudvīp, étant celui des hommes.

16. C'est une condition de survie, pour les hommes et leurs troupeaux, de connaître les avantages et inconvénients des caractéristiques géographiques et orographiques. Le site d'un campement doit être abrité du vent par une colline ou un bosquet, situé sur un terrain pentu orienté vers le sud (au printemps la neige fond rapidement et la croissance de l'herbe est plus rapide), avec des points d'eau et des pâturages de qualité. La question de l'eau est essentielle, car les précipitations sont irrégulières et en quantité limitée. En hiver, les éleveurs choisissent des vallées protégées, offrant des abris relatifs ainsi que des congères dont la neige abreuve le bétail.

17. Pozdneev ([1887] 1978, p. 230) ; Mostaert (1956, p. 289). Comme l'astrologie, la géomancie est un trait d'union vivant entre le clergé et les laïcs.

18. Bla brang.

19. Le terme " géomancie » dans le contexte chinois est aujourd'hui contesté, car cette pratique déborde largement la technique divinatoire en raison de ses aspects intellectuels et sociaux. Les anglophones lui préfèrent le terme «siting ». Nous emploierons le terme moderne de fengshui, plus connu des Occidentaux que kanyu ou dili. Sur la théorie, voir Feuchtwang (1974, pp. 172-203). Très à la mode aujourd'hui tant en Chine qu'en Occident, le fengshui a été récemment (ré)importé en république de Mongolie. Voir l'article de Grégory Delaplace dans le présent volume. 
20. Ces géomanciens sont parfois des religieux bouddhistes ou taoïstes. Sur cette profession, voir smith (1991, pp. 131-171, notamment p. 148 sq.).

21. Les charpentiers travaillaient sous l'autorité des lamas, qui possédaient des connaissances de base en architecture et recrutaient la main-d'œuvre localement.

22. On connait également un livre chinois qui contient quelques remarques en mongol: Dili wujue quanba ben [Les cinq secrets de la géomancie] - il était destiné à être traduit en mongol, mais n'a pas été complété.

23. Aүula ba nutuү-un sinzi-jin bicig [Les caractéristiques des montagnes et des pâturages] et Ene anu yazar-un sinzin bolai [Voici les caractéristiques du terrain] : Heissig (1971, pp. 148-151). Ces deux manuels ainsi que les deux précédents proviennent certainement de Mongolie-Intérieure.

24. Ce texte, destiné plutôt aux éleveurs, commence par une formule d'invocation à Lokeśvara, maître du sol, et au saint Mañjuśrī, bodhisattva de la connaissance et protecteur de la divination. Il préconise de pratiquer la méditation surMañjuśrī, d'être yogācārin, ou d'avoir connu l'essence du vide en méditation. "Si les chercheurs de site n'agissent pas ainsi, ils échoueront » (sarközi 1976, pp. 591-592 et 594-595).

25. Srong btsan sgam po.

26. Cf. le texte traduit par sarközi (1976, p. 591), et Mong. 314 de la Bibliothèque royale de Copenhague. Les Tibétains rattachent la géomancie aux sciences divinatoires qui seraient venues de Chine; mais la géomancie a également reçu des influences indiennes et subi des transformations proprement tibétaines (Aris 1979, pp. 6, 12-15, 20-21).

27. Un des responsables du centre de fengshui d'Oulan-Bator, fondé en mars 2002, pense que la géomancie est apparue il y a 4000 ans; il ne connaît pas son pays d'origine, mais lie son émergence à celle du bouddhisme. "Le fengshui, par la suite, est passé par la Chine, le Tibet, et l'Europe, avant d'arriver en [république de] Mongolie en mars 2002 » (Grégory Delaplace, communication personnelle, 2002).

28. Cf. l'ouvrage de Thubten Legshay Gyatsho (né en 1920), abbé du nouveau temple de Lumbini. Ce traité, traduit en anglais sous le titre Gateway to the Temple (1979),qui distille de nombreux textes anciens, est aujourd'hui un manuel de référence pour la construction et la décoration modernes de temples du bouddhisme tibétain (voir pp. 29-33 pour la géomancie). $C f$. également Meyer et Jest (1987, pp. 152-153 et p. 166 note 8) et Bawden (1961b, pp. 228-229 note 43).

29. Cf. les quatre « piliers » et les quatre « protecteurs » de la terre dans la géomancie tibétaine : le tigre clair à l'est, matérialisé par un chemin étroit ou un rocher, etc. (Thubten... 1979, p. 29). Ces caractéristiques, en particulier l'association d'un animal à une couleur et à un orient, sont probablement inspirées, avec quelques modifications, du fengshui (Aris 1979, p. 21 ; Feuchtwang 1974, pp. 151-158).

30. Le terme mandala, qui signifie en sanskrit " cercle ", désigne toutes sortes de représentations cosmologiques, et plus spécifiquement le palais céleste d'une divinité entouré de «cercles de protection » et servant de support de méditation. Le méditant "pénètre » en esprit dans les différentes enceintes du mandala par la porte est et chemine vers le centre dans le sens de la circumambulation rituelle. Pour une synthèse de travaux récents sur lemandala, voir stoddard (1999).

31. L'architecture bouddhique établit une série de correspondances entre le microcosme et le macrocosme, entre le corps de Bouddha, le panthéon divisé en cinq familles de bouddhas associées chacune à une région de l'espace (les quatre points cardinaux et le zénith), lemandala, et le stūpa, orienté et organisé autour d'un axe central.

32. Le fondateur du Badyar coiling süm-e (M.-I.) abandonna un site après quelques années, parce que les chèvres dévoraient la moindre pousse de céréales. Longtemps après, il vit un aigle transportant une couronne; il suivit ce signe de très bon augure et établit sa communauté à l'endroit où l'aigle posa l'objet (chuanZengxiang 1941, juan 13). Le géomancien chinois qui 
détermina l'emplacement $d u$ monastère de Šongqur vit s'envoler un faisan puis aperçut un serpent, il interpréta cette « conjoncture du vol du dragon et de la danse du phénix » comme un signe de bon augure (Erihen1986, p. 79). Le fondateur du Гaqai-jin süm-e («monastère du cochon »), dans la bannière des Zarud (ligue de Zirim, M.-I.) vit un jour sur la pente sud du mont Darqan un cochon blanc qui s'enfuit, tenant dans son groin des joyaux. Il décida de construire un monastère sur le territoire que ce cochon blanc avait purifié (Kürelša 1993, p. 23).

33. Pour l'organisation des bâtiments monastiques dans l'ensemble du monde bouddhique, voir pichard et Lagirarde (2003).

34. De même dans l'architecture tibétaine. L'architecture chinoise, tant religieuse que profane, place les édifices principaux (temple, résidence de l'abbé, salle du dharma pour un monastère bouddhique) « au fond »; en revanche, elle valorise le côté est par rapport au côté ouest.

35. L'orientation est importante dans la yourte: des axes de genre et d'aînesse conditionnent l'emplacement du mobilier, la place de chacun, les attitudes corporelles et la répartition des activités domestiques. L'espace intérieur (avant/arrière, gauche/droite) reflète une hiérarchie spatiale qui valorise le fond - le qoimar, espace honorifique réservé au chef de famille et aux hôtes de marque où se trouve l'autel - par opposition à la moitié sud laissée aux femmes et aux jeunes. Une deuxième division nord/sud sépare la moitié droite (ouest lorsque la yourte ouvre au sud) masculine, plus valorisée, de la moitié gauche féminine.

36. Cf.Nagao ([1947] 1991, pp. 148-150), ŠČepetil'nikov (1960, pp. 89-111) et Maidar (1972, pp. 20-42) - classification reprise par miller (1959, pp. 34-35) et Alexandre (1993, p. 84).

37. Les Tibétains conçoivent trois mondes étagés, qui se reflètent dans l'élévation de leur maison : le monde souterrain des klu (divinités chtoniennes aquatiques), le monde des hommes et le monde des dieux, reliés par une échelle (stein 1987, p. 188 sq.).

38. Cette disposition, héritée des camps de guerre de l'empire gengiskhanide, s'est perpétuée dans les campements de l'armée impériale mandchoue, ainsi que dans les campements mongols d'époque Qing : voir la disposition et l'orientation du camp d'été du prince des Abaya de l'Est au $\mathrm{XX}^{\mathrm{e}}$ siècle dans Jagchid et Hyer (1978, p. 67). Des dessins idéalisés et fantaisistes du XIX ${ }^{\mathrm{e}}$ siècle représentent les capitales mogholes telle Lahore comme un camp formé de plusieurs rectangles concentriques (Gaborieau 1995, p. 22).

39. Cf.wasilewski (1976, p. 355), citant des chants bouddhiques, et les peintures du XIX $x^{e}$ siècle représentant Jeke Kürij-e au musée des Beaux-Arts et au Palais Vert d'Oulan-Bator.

40. En Asie du Sud et du Sud-Est, les monastères à plan carré de Pagan en Birmanie ont également choisi d'éviter tout marquage du centre (Pierre Pichard, communication personnelle).

41. L'erüke laisse également passer les rayons du soleil, dont la position dans la yourte permet aux Mongols de connaître le moment de la journée, et donc de se repérer dans le temps. De même que le foyer et le ou les poteau(x), l'anneau de compression en forme de roue (toyunu) formant l'ouverture de l'erüke a une forte charge symbolique et rituelle, liée à la fécondité, la richesse et la circulation de la force vitale. C'est par l'orifice à fumée que le chamane bouriate quitte la yourte dans son voyage spirituel et que descend la prospérité sur la famille.

42. Sur le symbolisme du lanterneau, voir stein (1987, pp. 131 et 150-151).

43. Par exemple, la salle d'assemblée du Zegün yar zuu (dans la bannière Zegün yar, ligue des Ordos) coiffée d'une grande toiture à versants; ou encore les grands coycin en bois à plan carré de l'ancienne Ourga, dérivés de la yourte, et qui pouvaient atteindre des tailles considérables; et également les coycin surmontés d'étages en réduction des temples de Bouriatie.

44. Les anciens temples-mandala tibétains comme Samye (bSam-yas) ont pourtant été influencés par le mingtang ("palais de lumière », maison du calendrier répliquant l'univers) de la Chine ancienne. 
45. La circumambulation est effectuée dans le sens des aiguilles d'une montre, la main droite " pure » étant du côté de l'objet sacré. Pour certaines divinités tutélaires comme celles des tantramères, la circumambulation doit se faire dans l'autre sens (stoddard 1999).

46. La circumambulation se dit encore dharmacakrapravartana, «mise en branle de la roue de la loi ».

47. L'Histoire secrète des Mongols relate la ronde autour de l'Arbre-Touffu (Even et Pop 1994, § 57 et p. 262 note 118). Les Bouriates connaissent des danses similaires suivant le « chemin du soleil ».

48. Le mouvement dans le sens de la marche du soleil est associé à ce qui est droit et garantit l'accroissement de force vitale ; le mouvement inverse est associé à la distorsion, à la stagnation, au chaos, à la mort. On découvre l'orifice à fumée de la yourte, on bouge dans la yourte dans le sens des aiguilles d'une montre; l'ordre de montage et de démontage de la yourte, de même que tout mouvement circulaire effectué pendant ces opérations, suit le mouvement du soleil en commençant par l'ouest de la porte, car le montage symbolise la création du monde (chabros et Dulam 1990, p. 23). Il est courant, avant d'entrer dans un endroit particulièrement respecté, d'en faire le tour extérieur dans le sens des aiguilles d'une montre. Lors du rituel de la première traite des juments au début de l'été, on tourne autour de la corde (zele), et, pour le rituel d'appel de la bonne fortune (dalaly-a), on décrit des mouvements circulaires en tenant une flèche et on fait parfois tourner le bétail autour de la yourte toujours dans ce même sens. La circumambulation se pratique également pendant d'autres rituels : érection d'une nouvelle yourte, mariage, sacrifice à l'obo, nayadum (qalq-a : naadam),etc. (voir pegg2001, pp. 93, 178, 182, 195 et 289). En revanche, on n'a pas coutume en Mongolie de faire le tour des montagnes sacrées, comme c'est l'usage lors des pèlerinages tibétains.

49. bKa' 'gyur.

50. De tels aménagements architecturaux à cette époque surprennent, car au Tibet on ne construisait déjà plus ces couloirs, et ce dès le $\mathrm{xv}^{\mathrm{e}}$ siècle pour le Tibet central.

51. Pour le pavillon Alaybaiba qu'il fit construire en Mongolie Qalq-a(probablement à Jeke Kürije), Zanabazar demanda qu'une porte soit construite au nord-est de la salle d'assemblée, aménagement qui parut alors inutile. Sous le II Zibcündamba qutuүtu, un moine décéda dans la salle d'assemblée pendant le service et le qutuүtu ordonna de lever le rideau au nord-est (on avait donc caché cette porte) et de sortir le moine par là. Puis il dit de supprimer cette porte et de rendre «complet l'intérieur et l'extérieur du pavillon " (Bawden 1961a, p. 63, folio 22r, et p. 74, folio $30 \mathrm{v}$ ). Ceci montre le caractère anormal de ce type de porte, justifié néanmoins par les croyances chamaniques : les morts et les vivants, les hommes et les esprits/les chamanes, n'utilisent pas les mêmes entrées. Si quelqu'un meurt dans une yourte, on a coutume de faire sortir le corps par une ouverture pratiquée en enlevant une section du treillis.

52. Le plan de Shangdu (Kaiping), capitale fondée par Qubilai Qan en 1256-1259, est basé sur les Huit trigrammes du Yijing et sur un mandala (avec huit monastères bouddhiques aux quatre points cardinaux et aux quatre angles). Le plan du Pékin des Yuan (Dadu ou Qanbaliq) utilisa également la numérologie des Huit trigrammes, ainsi que le plan chinois classique idéal, centré et orienté, de la capitale des Zhou tel qu'il est décrit dans un célèbre traité de construction, le Kaogong ji. Des légendes mongoles décrivent encore la construction de Dadu comme un mandala du dieu Nata (chinois : Nezha).

53. En particulier, stein $(1987,2 \S$ partie. L'habitation, aspects réels), wasilewski (1976), chabros (1988),chabros et Dulam (1990), Tangad (1990), sauer (2001), etc.

54. La cosmologie mongole ancienne est essentiellement binaire; elle oppose, dans une relation mêlant orientation, séniorité et couleur, les 55 tengri de l'Ouest (aînés, blancs) aux 44 de l'Est (cadets, noirs) - le nord et le sud restant des « orients vides" (Hamayon 1978, p. 223). Roberte Hamayon (1990, pp. 708-715) a démontré la verticalisation de la conception de l'espace chez les éleveurs, dans une logique de filiation, par rapport à la perception horizontale des chasseurs, 
dans une logique d'alliance. Toutefois, cette verticalisation se traduit dans l'orientation spatiale par une opposition entre l'amont et l'aval de la rivière, et non par l'entrée en jeu d'une troisième dimension perpendiculaire. On ne peut donc parler de vision étagée du monde comme chez les Tibétains : les nomades mongols ne considéraient pas la montagne comme un axe du monde et le corps comme un microcosme avant l'influence de la cosmologie bouddhique. D'ailleurs, les « trois mondes » sont différents dans la vision mongole "populaire », qui n'a adopté que partiellement les notions bouddhiques. Le monde souterrain, gouverné par Erlig qan, est aquatique, mais n'est pas la demeure des âmes défuntes, et à la montagne, bénéfique, sont opposées les eaux, maléfiques (Hamayon 1990, pp.709-715). Certains auteurs parlent d'une organisation étagée, tridimensionnelle, de la yourte qui contiendrait trois mondes reliés par l'axis mundi (le ou les poteaux), tels tangad (1990) et chabros (1988, p. 34). Cette représentation, de même que les notions de centre, d'ouverture supérieure, de pilier-pivot, d'étoile polaire qui soutient la voûte céleste et de "mandala » fondés sur le chiffre neuf, témoigne d'influences chinoises ou d'un fonds commun sino-mongol très ancien (stein 1987, p. 120 sq. ; snodgrass 1990, pp. 331-392 ; wasilewski 1976, pp. 354-357), ainsi que d'influences bouddhiques.

55. Ainsi le nord est-il tantôt source de richesses - on considère par exemple que la grâce (kesig) vient du nord de la tente vers ses habitants (chabros 1988, p. 32) -, tantôt néfaste. Le nord-est serait aussi néfaste (Even 1988-1989, p. 179 note 8).

\section{RÉSUMÉS}

L'orientation revêt-elle une importance symbolique particulière dans l'architecture des monastères bouddhiques mongols fondés entre le $\mathrm{xvI}^{\mathrm{e}}$ et le $\mathrm{xx}^{\mathrm{e}}$ siècle ? Dans le choix de leur site par des pratiques géomantiques, leur inscription dans le paysage, l'orientation générale de leurs bâtiments, la hiérarchie entre différentes positions (droite et gauche, haut et bas, entrée et fond), leur plan d'ensemble et la structure de leur temple principal se décèlent influences bouddhiques indo-tibétaines, influences chinoises et conception mongole de l'espace. Temples axiaux, temples-mandala et stūpa renvoient à une progression axiale ou à des modèles cosmologiques centrés et orientés. Un plan ou une implantation, peu "ordonné ", non conforme à un modèle cosmologique sera rectifié dans les représentations littéraires ou picturales, et il est instructif d'étudier l'écart entre discours et réalité. En dernier lieu est évoquée la pratique de la circumambulation, qui unit en un même mouvement parcours spatial et parcours temporel.

The article treats the question of whether orientation becomes particularly important in the architecture of Mongolian Buddhist monasteries built between the 16th and the 20th century. While the monasteries were oriented, the orientation was multiple: Indo-Tibetan Buddhist influences, Chinese influences, and the Mongolian conception of space all played contrasting roles in the choices of the right setting (by means of geomancy), the monument's inscription in the landscape, the buildings' general orientation, the hierarchy between different positions (right and left, high and low, entrance and back), and their general layout. As a consequence, various models coexist: axial temples, mandala temples and stūpa are built according to either an axial progression or centred and oriented cosmological models. Furthermore, some sites are built in an apparently disorderly layout or settlement, which bears little resemblance to a cosmological model. Yet, such sites will be "corrected" in literary or pictorial representations, by 
evincing telling discrepancies between the discourse and the reality of orientation.Finally we discuss the practice of circumambulation which unites spatial and temporal progressions.

\section{INDEX}

Index géographique : Mongolie, Mongolie Intérieure

Thèmes : architecture, bouddhisme, cosmologie, orientation nomsmotscles Halh

Keywords : Buddhist monastery, circumambulation, geomancy, mandala, spatial organization, stupa, symbolism

Mots-clés : circumambulation, géomancie, mandala, monastère bouddhique, organisation spatiale, stupa, symbolisme

\section{AUTEUR}

\section{ISABELLE CHARLEUX}

Isabelle Charleux est chargée de recherche au C.N.R.S. dans le Groupe de Sociologie des Religions et de la Laïcité (C.N.R.S.-E.P.H.E.). Ses recherches portent sur la culture matérielle des Mongols, l'art et l'architecture du bouddhisme tibéto-mongol en Chine. 